DrAft VERSION OCTOBER 12, 2018

Preprint typeset using $\mathrm{IAT}_{\mathrm{E}} \mathrm{X}$ style emulateapj v. 5/2/11

\title{
APPLICATION OF GAS DYNAMICAL FRICTION FOR PLANETESIMALS: II. EVOLUTION OF BINARY PLANETESIMALS
}

\author{
Evgeni Grishin \& Hagai B. Perets \\ Physics Department, Technion - Israel Institute of Technology, Haifa, Israel 3200003 \\ Draft version October 12, 2018
}

\begin{abstract}
One of first the stages of planet formation is the growth of small planetesimals and their accumulation into large planetesimals and planetary embryos. This early stage occurs much before the dispersal of most of the gas from the protoplanetary disk. At this stage gas-planetesimal interactions play a key role in the dynamical evolution of single intermediate-mass planetesimals $\left(m_{p} \sim 10^{21}-10^{25} \mathrm{~g}\right)$ through gas dynamical friction (GDF). A significant fraction of all Solar system planetesimals (asteroids and Kuiper-belt objects) are known to be binary planetesimals (BPs). Here, we explore the effects of GDF on the evolution of binary planetesimals embedded in a gaseous disk using an N-body code with a fiducial external force accounting for GDF. We find that GDF can induce binary mergers on timescales shorter than the disk lifetime for masses above $m_{p} \gtrsim 10^{22} \mathrm{~g}$ at $1 \mathrm{AU}$, independent of the binary initial separation and eccentricity. Such mergers can affect the structure of merger-formed planetesimals, and the GDF-induced binary inspiral can play a role in the evolution of the planetesimal disk. In addition, binaries on eccentric orbits around the star may evolve in the supersonic regime, where the torque reverses and the binary expands, which would enhance the cross section for planetesimal encounters with the binary. Highly inclined binaries with small mass ratios, evolve due to the combined effects of Kozai-Lidov cycles with GDF which lead to chaotic evolution. Prograde binaries go through semiregular Kozai-Lidov evolution, while retrograde binaries frequently flip their inclination and $\sim 50 \%$ of them are destroyed.
\end{abstract}

\section{INTRODUCTION}

Planets form in protoplanetary disks around young stars. Once $\mathrm{km}$ sized planetesimals have been formed, their evolution is determined by three basic dynamical processes: Viscous stirring, dynamical friction and coagulation or disruption through collisions (see Goldreich et al. 2004 and references therein for details). These dynamical processes do not include the effects from planetesimal-gas interaction in the disk, which can be important during the early stages of planet formation when gas is abundant (the first few Myr, with possible suggestions for longer timescales (Pfalzner et al. 2014).

During the evolutionary phases of planet formation, small dust grains successively grow into large planetary embryos. The evolution of small size planetesimals is dominated by gas drag. Gas drag keeps the planetesimal disk thin, the relative velocities low, and assists in coagulation of small bodies (Ormel \& Klahr 2010; Ohtsuki et al. 2002; Perets 2011). Larger planetary embryos (i.e. $m_{p} \geq 0.1 M_{\oplus}$ ) are also affected by gas interactions, and can migrate through type I or type II migration (see Papaloizou \& Terquem 2006 and references therein).

In our previous paper (Grishin \& Perets 2015; hereafter; paper I), we have studied the planetesimal-disk interactions of intermediate mass planetesimals (IMP), in the mass range $10^{21} \leq m_{p} \leq 10^{25} \mathrm{~g}$, using the gas dynamical friction (GDF) approach, and found it to be efficient in changing the orbital evolution of the IMPs, and significantly more important than aerodynamical gas drag in this mass range.

The interaction between a gravitating planetesimal and the nebular gas was first studied by Ohtsuki et al. (1988). In their study, the effective drag coefticient increases rapidly with increasing gravitational energy. The first detailed analytic study of GDF was by Ostriker (1999) for objects in homogenous environment moving on straight line. Kim \& Kim (2007) have generalized Ostriker s work for circular orbits. GDF for binary perturbers was first introduced by Kim et al. (2008), and Sánchez-Salcedo \& Chametla (2014) who studied the evolution of a binary traveling in a non-stationary gaseous medium, generalizing further previous works. Here, we focus on the effects of GDF on binary planetesimals (BPs) which had not been explored before, and complement previous work on the evolution of lower mass BPs due to aerodynamical gas drag (Perets \& Murray-Clay 2011).

Paper I dealt with strictly single planetesimals. However, observations indicate that a large fraction (at least $20 \%$ ) of all Kuiper Belt and Trans Neptunian Objects (KBOs, TNOs) are gravitationally bound binaries (Noll 2007). Such binaries could play an important role in planet formation and significantly accelerate planetesimal growth (Perets 2011; Richardson \& Walsh 2006 and references therein). Goldreich et al. (2002) showed that massive BPs could be formed through dynamical friction due to small planetesimals; a transient binary becomes bound after losing its energy during an encounter due to dynamical processes, and is then hardened by collisionless dynamical friction due to small bodies. They find that $\sim 5 \%$ of Kuiper Belt Objects could form bound binaries through such mechanism. Nesvorný et al. (2010) suggested that BPs can from through a gravitational collapse, and Kominami et al. (2011); Kominami \& Makino (2014) explored the dynamical formation of BPs.

In paper I we found that GDF damps inclinations and eccentricities of planetesimal orbits around the Sun efficiently even if the mass is as low as $\sim 10^{23} \mathrm{~g}$, given that 
the random velocity is not too high $\left(e, I \sim\right.$ few $\times H_{0}$, where $e$ and $I$ are the orbital eccentricity and inclination respectively, $H_{0}=h / a$ is the aspect ratio, $h$ is the disk scale height). In addition, large planetary embryos of mass $\sim 10^{25} \mathrm{~g}$ migrate within a few Myrs. One of the implications of IMP interaction with the gas is the cooling of the planetesimal disk, and the potential overabundance of IMPs in the inner region, that could potentially assist the formation of Super-Earths (see discussion in paper I).

Motivated by recent developments both in GDF theory for binaries and planet formation, we extend the study of paper I on intermediate mass binary planetesimals (BPs), and show that GDF can change the binary mutual orbit, either leading to binary inspiral and even merger or to the expansion of the binary separation, depending on the properties of the binary orbit around the Sun.

The paper is organized as follows: In section 2 we review the gas-planetesimal interactions. In section 3 we discuss the limitations and applicability of our study. In section 4 we derive the differential accelerations of BPs due to GDF. In section $\mathbf{5}$ we use the differential accelerations and derive analytic expressions for the evolutionary timescales of binary orbital elements. In section $\mathbf{6}$ we present the numerical set up and the initial conditions of the tested scenarios. We present the numerical results in section 7. In section 8 we discuss the significance of our results. Finally, we summarize the paper in section $\mathbf{9}$.

\section{GAS PLANETESIMAL INTERACTIONS}

The standard models of evolution of planetesimal disks account for various dynamical processes, including both physical processes due to planetesimal-planetesimal interactions such as viscous stirring, dynamical friction and physical collisions followed by coagulation, as well as gasplanetesimal interactions through aerodynamics gas-drag on small planetesimals, and planetary migration through tidal torques acting on large planetary embryos and planets.

Although gas-planetesimal interaction has been studied in the context of small planetesimals, massive planetesimals have been largely ignored as they are decoupled from the gas, and their size and velocity dispersion is dominated by gravitational interactions. In paper I we have studied IMPs in the mass range $10^{21}-10^{25} \mathrm{~g}$ and explored their interactions with the gas through GDF, which dominate over aerodynamics gas drag in this mass range. In the following we briefly review the GDF approach and its comparison to aerodynamic gas drag. More details can be found in paper I.

The general aerodynamic drag force imposed on a planetesimal of radius $R$ and relative velocity $v_{r e l}$ moving through a gaseous medium of density $\rho_{g}$ is

$$
\boldsymbol{F}_{D}=-\frac{1}{2} C_{D} \pi R^{2} \rho_{g} v_{r e l}^{2} \hat{\boldsymbol{v}}_{r e l}
$$

where $\hat{\boldsymbol{v}}_{\text {rel }}$ is the unit vector in the direction of the relative velocity and $C_{D}$ is the drag coefficient that for spherical body depend on the Reynolds number, i.e. $C_{D}=$ $C_{D}(\mathcal{R} e)$. Empirical formula can be used for $C_{D}(\mathcal{R} e)$, fitted for the range $\log _{10} \mathcal{R} e \in[-3,5]$ (see.Brown \& Lawyer 2003 and paper I).

For the GDF force, consider a perturber with mass $m_{p}$ moving on a straight line with the same ambient gaseous medium. The perturber generates a wake, which in turn affects the perturber. Using linear perturbation theory, Ostriker (1999) calculated the drag force felt by the perturber. The GDF force is given by

$$
\boldsymbol{F}_{G D F}=-\frac{4 \pi G^{2} m_{p}^{2} \rho_{g}}{v_{\text {rel }}^{3}} \boldsymbol{v}_{\text {rel }} \mathcal{I}(\mathcal{M}),
$$

where $\mathcal{M} \equiv v_{\text {rel }} / c_{s}$ is the Mach number, $c_{s}$ is the speed of sound and $\mathcal{I}(\mathcal{M})$ is a dimensionless factor given by

$\mathcal{I}(\mathcal{M})=\left\{\begin{array}{cc}\frac{1}{2} \ln \left(\frac{1+\mathcal{M}}{1-\mathcal{M}}\right)-\mathcal{M} & \mathcal{M}<1 \\ \frac{1}{2} \ln \left(1-\mathcal{M}^{-2}\right)+\ln \left(\frac{v_{\text {rel }} t}{R_{\text {min }}}\right) & \mathcal{M}>1 ; v_{\text {rel }} t>R_{\text {min }}\end{array}\right.$

where $\ln v_{\text {rel }} t / R_{\text {min }}$ is the Coulomb logarithm. The force is non-vanishing in the subsonic regime, while in the supersonic regime, a minimal radius $R_{\text {min }}$ is introduced to avoid divergence of the gravitational potential (usually taken to be the physical size of the perturber, or the accretion radius $\left.G m_{p} / v_{r e l}^{2}\right)$. The exact value of $R_{m i n}$ is not well determined; but it can be fitted through comparison of eqn. (3) with hydrodynamical simulations, to find a best fitting value for $R_{\min }$ (Sánchez-Salcedo \& Brandenburg 1999). The maximal scale of the system is usually the disk scale height $h$, so typical values of the Coulomb logarithm are near $\sim 10$.

We note that the results of Kim \& Kim (2007) are qualitatively similar to Ostriker s straight line trajectory (see fig. 8 of Kim \& Kim 2007).

Another useful formula for the subsonic regime is the expansion in powers of Mach number

$$
\mathcal{I}(\mathcal{M})=\frac{1}{3} \mathcal{M}^{3}+\frac{1}{5} \mathcal{M}^{5}+\mathcal{O}\left(\mathcal{M}^{7}\right) .
$$

If only the first term is considered, the GDF force scales linearly with $\boldsymbol{v}_{r e l}, \boldsymbol{F}_{G D F} \propto-\boldsymbol{v}_{r e l}$, and thus we will occasionally use the term 'linear regime' for the subsonic regime.

In paper I we found the critical planetesimal size $R_{\star}$ that GDF equals to aerodynamic gas drag. It is given by

$$
R_{\star}=0.29\left[\frac{C_{D}(\mathcal{R} e)}{\mathcal{I}(\mathcal{M})}\right]^{1 / 4} \frac{v_{r e l}}{\sqrt{G \rho_{m}}},
$$

where $v_{r e l} \approx \sqrt{H_{0}^{4}+e^{2}} v_{K}, v_{K}=\sqrt{G M_{\star} / a}$ is the Keplerian velocity, $a$ is the semi-major axis, and $e$ is the orbital eccentricity.

In paper I we used a simple flared disk model (Chiang \& Goldreich 1997) and found that typical masses where GDF' dominates over aerodynamical gas-drag are $\gtrsim 10^{21} \mathrm{~g}$. The parametric dependence of type I migration and GDF torque is the same, and GDF is comparable to type I one sided torque. The tidal torque is a superposition of inner and outer torques, and is smaller by a factor of $\sim h / a$ due to the asymmetry of the inner and outer regions of the disk (see paper I).

\section{FORMULATION OF THE PROBLEM, ASSUMPTIONS AND APPLICABILITY}

The effect of GDF for binary perburbers was studied in detail in Kim et al. (2008). When considering the wakes of both of the bodies, the azimuthal component of 
GDF, $F_{\varphi} / \mathcal{M}^{2}$ was reduced due to the companion's wake. For low Mach numbers, $F_{\varphi} / \mathcal{M}^{2}$ was negligible, and for $\mathcal{M} \sim 1$ it was $\sim 30 \%$ less. However, the latter is true for static gas. Consider a binary system that the center of mass $(\mathrm{CM})$ of the binary revolves around the central object (e.g. Earth-Moon system that revolves around the Sun. The inner system is considered as the inner binary (e.g. Earth-Moon system), while the combination of the central object with the CM of the inner binary is considered as the outer binary (e.g. Sun - CM of Earth-Moon system). If the outer binary moves with relative velocity $\boldsymbol{v}_{\text {out }}$ with respect to the gas, the morphology of the wake is different. Sánchez-Salcedo \& Chametla (2014) have shown that the braking torque that causes the binary to lose angular momentum, is $\Gamma=\alpha a_{b i n} F_{D G F, \varphi}^{(1)}$ where $F_{D G F, \varphi}^{(1)}$ is the GDF force applied on the first body, $a_{b i n}$ is the binary separation, and $\alpha$ is an order of unity parameter that depends on the minimal radius $R_{\text {min }}$; the primary Mach number is $\mathcal{M}_{c m}=v_{\text {out }} / c_{s}$; and the binary Mach number is $\mathcal{M}_{b i n}=v_{b i n} / c_{s}$, where $v_{b i n}$ is the typical binary velocity given by

$$
v_{b i n}=\sqrt{\frac{G m_{b i n}}{f r_{H}}}=v_{K} f^{-1 / 2} Q^{1 / 3} .
$$

In principle, the function $\alpha\left(R_{\text {min }}, \mathcal{M}_{c m}, \mathcal{M}_{\text {bin }}\right)$ can be calculated numerically for each case. It has been calculated by Sánchez-Salcedo \& Chametla (2014) for some cases but the full parameter space has not been explored. Nevertheless, it is of order unity and $<1$ for most cases.

We do not account for the companion's wake in our simulation. By doing that, we overestimate GDF force by at most $2-3$ times, according to both Kim et al. (2008) and Sánchez-Salcedo \& Chametla (2014). Moreover, for typical planetesimal masses and for eccentric primary orbit, $v_{\text {out }} \gg v_{\text {bin }}$. In this regime $\alpha \rightarrow 1$ and the force is correctly estimated.

In paper I we studied the effects of GDF on single planetesimals. Here we extend our study to BPs.

Let us consider a binary with separation of the order of the binary mutual Hill radius, $r_{H}$, i.e. $a_{b i n}=f r_{H}=$ $f a_{\text {out }}\left(m_{\text {bin }} /\left(m_{\text {bin }}+M_{\star}\right)\right)^{1 / 3} \approx a_{\text {out }} f Q^{1 / 3}$ where $Q \equiv$ $m_{\text {bin }} / M_{\star}$ is the star-binary mass ratio, $m_{\text {bin }}=m_{b}+m_{s}$ is the total binary mass, $a_{\text {out }}$ is the semi-major axis of the center of mass $(\mathrm{CM})$ of the binary to the central star and $0<f<1$ is some fraction. Usually the limit of stable prograde orbits is near $f \approx 0.5$, while the limit of retrograde orbits is twice as large (Hamilton \& Burns 1991). Note that $r_{H}$ is an additional length scale in the system, which is important for the applicability of GDF to BPs.

The density wave propagating in $\hat{\boldsymbol{z}}$ direction reaches the disk scale height after $\sim 1$ orbital period and GDF is gradually suppressed. In order to tackle the problem, Muto et al. (2011) solved the equations of motion in a slab geometry. They introduced an averaged potential at the scale height of the disk. Using Fourier analysis, they have shown that the dominant contributions are from perturbations with length scale as the disk scale height $l \sim h$. While it is marginally applicable for single planetesimals (e.g. one needs to invoke the Kolmogorov description of turbulence of the gaseous disk; see discus- sion in paper I), here the contributions to the perturbation on the mutual binary orbit are much less than the scale height, since the relevant scale height is the binary Hill radius which is smaller than the scale height of the disk. Ostriker (1999)'s 3D analysis remains valid as long as $r_{H}<h$, or $Q^{1 / 3}<(h / a)^{3}$. With $h / a \sim 0.022$ in our case, the restriction is $Q \lesssim 10^{-5}$. Hence, only for masses of $\approx 4 M_{\oplus}$ and above do 3D modeld break down. In our simulations the highest masses of planetesimals are $m_{p} \lesssim 2 \cdot 10^{25} \mathrm{~g}$, so we are well within the thick disk regime.

To conclude, Ostriker (1999)'s 3D formalism has difficulties describing GDF for single planetesimals, but it fits well for describing the mutual binary orbits for planetesimals of mass $m_{b i n} \lesssim 4 M_{\oplus}$. As long as the the binary mass is much lower than Earth mass, we can consider the binary to be embedded in a spherical gaseous halo.

\section{DIFFERENTIAL ACCELERATIONS}

Similarly to the effects of aerodynamic gas drag on a binary planetesimal (Perets \& Murray-Clay 2011) differential force due to the interaction with the gas can shear apart the binary. In this section we calculate the differential acceleration. In appendix $\mathrm{A}$ we define the $G D F$ shearing radius and show that it is much larger than the Hill radius of the binary, thus concluding that BPs are stable to shearing effects by GDF.

Consider, for simplicity, a circular and co-planar binary with masses $m_{b} \geq m_{s}$ and total binary mass $m_{\text {bin }}=$ $m_{b}+m_{s}$. The magnitudes of the binary velocities of each body are $v_{b}=\left(m_{s} / m_{b i n}\right) v_{b i n}$ and $v_{s}=\left(m_{b} / m_{b i n}\right) v_{b i n}$, where the binary velocity is given in eq. 6. The relative velocities of each body with respect to the gas are

$\boldsymbol{v}_{b, r e l}=\boldsymbol{v}_{\text {out }}+\boldsymbol{v}_{b}=v_{\text {out }} \hat{\boldsymbol{\varphi}}\left(\nu_{\text {out }}\right)+\frac{m_{s}}{m_{\text {bin }}} v_{\text {bin }} \hat{\boldsymbol{\varphi}}_{\text {bin }}\left(\nu_{\text {bin }, b}\right)$,

and

$\boldsymbol{v}_{s, r e l}=\boldsymbol{v}_{\text {out }}+\boldsymbol{v}_{s}=v_{\text {out }} \hat{\boldsymbol{\varphi}}\left(\nu_{\text {out }}\right)+\frac{m_{b}}{m_{\text {bin }}} v_{\text {bin }} \hat{\boldsymbol{\varphi}}_{\text {bin }}\left(\nu_{\text {bin }, s}\right)$,

where $v_{\text {out }} \equiv \varepsilon v_{K}$ is the center of mass velocity, $\varepsilon \approx 3 H_{0}^{2}$ is the relative velocity scaling between the gas and a single planetesimal in a circular orbit (see paper I for details $) ; \hat{\varphi} \equiv\left(-\sin \nu_{\text {out }}, \cos \nu_{\text {out }}\right)$ is the unit vector of the binary's center of mass around the star; $\nu_{\text {out }}=\Omega_{\text {out }} t$ is the true anomaly of the primary; $\Omega_{\text {out }}=\sqrt{G M_{\star} / a_{\text {out }}^{3}}$ is the mean motion of the primary around the star; $\hat{\boldsymbol{\varphi}}_{\text {bin }}=$ $\left(-\sin \nu_{b i n}, \cos \nu_{b i n}\right)$ is the unit vector of the binary system; $\nu_{b i n}=\Omega_{b i n} t$ is the binary true anomaly; and $\Omega_{\text {bin }}=\sqrt{G m_{\min } /\left(f r_{H}\right)^{3}}$ is mean motion of the binary. Note that

$$
\Omega_{\text {bin }}=\sqrt{\frac{G m_{\text {bin }}}{f^{3} r_{H}^{3}}}=f^{-3 / 2} \Omega_{\text {out }} \gg \Omega_{\text {out }} .
$$

For circular binary, there is always a phase difference of $\pi$ in the binary's true anomalies, (i.e. $\mid \nu_{b i n, s}-$ $\left.\nu_{b i n, b} \mid=\pi\right)$, hence in Eqs. (7) and (8) $\hat{\varphi}_{\text {bin }}\left(\nu_{b i n, b}\right)=$ $-\hat{\varphi}_{b i n}\left(\nu_{b i n, s}\right)$. We chose the initial phase such that eqn. (7) has a minus sign in the second term, and omit the dependence on the true anomaly to simplify notation. 


\subsection{Linear force regime}

Consider the subsonic case, where the eccentricity of the center of mass (i.e. the primary), $e_{\text {out }}$ is small or zero. In this case, the relative velocities involved are small compared to the sound speed $c_{s}$, and the acceleration of each mass is linearly proportional to the velocity, i.e.

$$
\boldsymbol{a}_{G D F, i}=C_{s u b} m_{i} \boldsymbol{v}_{\boldsymbol{r e l}},
$$

where $C_{s u b}=4 \pi G^{2} \rho_{g} / 3 c_{s}^{3}$. The differential acceleration is

$$
\begin{aligned}
& \frac{\boldsymbol{\Delta} \boldsymbol{a}_{\boldsymbol{G} \boldsymbol{D} \boldsymbol{F}}}{C_{\text {sub }}}=m_{b} \boldsymbol{v}_{b}-m_{s} \boldsymbol{v}_{s} \\
= & m_{b}\left(\varepsilon v_{K} \hat{\boldsymbol{\varphi}}-\frac{m_{s}}{m_{b i n}} v_{b i n} \hat{\boldsymbol{\varphi}}_{\boldsymbol{b i n}}\right) \\
- & m_{s}\left(\varepsilon v_{K} \hat{\boldsymbol{\varphi}}+\frac{m_{b}}{m_{\text {bin }}} v_{\text {bin }} \hat{\boldsymbol{\varphi}}_{\boldsymbol{b i n}}\right) .
\end{aligned}
$$

Collecting terms proportional to either $\hat{\boldsymbol{\varphi}}$ or $\hat{\boldsymbol{\varphi}}_{\text {bin }}$ we get

$$
\frac{\Delta \boldsymbol{a}_{\boldsymbol{G} \boldsymbol{D} \boldsymbol{F}}}{C_{\text {sub }}}=m_{b}(1-q) \varepsilon v_{K} \hat{\boldsymbol{\varphi}}-2 \mu v_{\text {bin }} \hat{\boldsymbol{\varphi}}_{\boldsymbol{b i n}},
$$

where $\mu=m_{b} m_{s} /\left(m_{b}+m_{s}\right)=m_{s} /(1+q)$ is the reduced mass, and $q=m_{s} / m_{b}$ is the binary mass ratio.

\subsection{Supersonic regime}

If the eccentricity of the primary is larger than the aspect ratio, (i.e. $e_{\text {out }}>2 H_{0}$ ), the relative velocity of the primary $v_{\text {out }}$ is supersonic. In the supersonic regime, the acceleration of each body is proportional to $\boldsymbol{v}_{r e l} / v_{r e l}^{3}$, i.e.

$$
\boldsymbol{a}_{G D F, i}=C_{\text {super }}^{(i)} m_{i} \boldsymbol{v}_{\text {rel }} / v_{\text {rel }}^{3}
$$

where

$$
C_{\text {super }}^{(i)}=4 \pi G^{2} \rho_{g}\left(\ln \Lambda+\frac{1}{2} \ln \left(1-1 / \mathcal{M}_{i}^{2}\right)\right),
$$

with $\mathcal{M}_{i}$ being the instantaneous Mach number of body $i=b, s$. The second term in eqn. (14) is negligible compared to the Coulomb logarithm and can be omitted. Thus, $C_{\text {super }}^{(i)} \approx C_{\text {super }}$, where $C_{\text {super }}$ includes only the first term. Using the relative velocities in eqns. (7) and (8), the differential acceleration is

$$
\begin{aligned}
& \frac{\boldsymbol{\Delta} \boldsymbol{a}_{G D F}}{C_{\text {super }}}=m_{b} \frac{\boldsymbol{v}_{r e l, b}}{v_{r e l, b}^{3}}-m_{s} \frac{\boldsymbol{v}_{r e l, s}}{v_{r e l, s}^{3}} \\
= & m_{b} \frac{\varepsilon v_{K} \hat{\boldsymbol{\varphi}}-\left(m_{s} / m_{\text {bin }}\right) v_{\text {bin }} \hat{\boldsymbol{\varphi}}_{\text {bin }}}{\left|\varepsilon v_{K} \hat{\boldsymbol{\varphi}}-\left(m_{s} / m_{\text {bin }}\right) v_{\text {bin }} \hat{\boldsymbol{\varphi}}_{\boldsymbol{b i n}}\right|^{3}} \\
- & m_{s} \frac{\varepsilon v_{K} \hat{\boldsymbol{\varphi}}+\left(m_{b} / m_{\text {bin }}\right) v_{\text {bin }} \hat{\boldsymbol{\varphi}}_{\boldsymbol{b i n}}}{\left|\varepsilon v_{K} \hat{\boldsymbol{\varphi}}+\left(m_{b} / m_{\text {bin }}\right) v_{\text {bin }} \hat{\boldsymbol{\varphi}}_{\boldsymbol{b i n}}\right|^{3}} .
\end{aligned}
$$

In order for the supersonic regime to apply for mass range of planetesimals we consider, the primary orbit must be sufficiently eccentric or inclined. For large $e_{\text {out }}$, the relative velocity of the primary is dominated by the eccentricity. In this case $\varepsilon \approx e_{\text {out }}$. It is convenient to define $\beta=v_{\text {bin }} / \varepsilon v_{K} \ll 1$ to get

$$
\begin{aligned}
\frac{\boldsymbol{\Delta} \boldsymbol{a}_{G D F}}{C_{\text {super }}} & =m_{b} \frac{e_{\text {out }} v_{K}\left(\hat{\boldsymbol{\varphi}}-\left(m_{s} / m_{\text {bin }}\right) \beta \hat{\boldsymbol{\varphi}}_{\text {bin }}\right)}{e_{\text {out }}^{3} v_{K}^{3}\left|\hat{\boldsymbol{\varphi}}-\left(m_{s} / m_{\text {bin }}\right) \beta \hat{\boldsymbol{\varphi}}_{\text {bin }}\right|^{3}} \\
& -m_{s} \frac{e_{\text {out }} v_{K}\left(\hat{\boldsymbol{\varphi}}+\left(m_{b} / m_{\text {bin }}\right) \beta\right) \hat{\boldsymbol{\varphi}}_{\text {bin }}}{e_{\text {out }}^{3} v_{K}^{3}\left|\hat{\boldsymbol{\varphi}}+\left(m_{b} / m_{\text {bin }}\right) \beta \hat{\boldsymbol{\varphi}}_{\text {bin }}\right|^{3}} .
\end{aligned}
$$

In appendix $\mathrm{B}$ we show that to first order in $\beta$, eqn. 16 can be written as

$\frac{\boldsymbol{\Delta} \boldsymbol{a}_{G D F}}{C_{\text {super }}}=\frac{m_{b} e_{\text {out }} v_{K}(1-q) \hat{\boldsymbol{\varphi}}+3 q m_{b} v_{\text {bin }} \hat{\boldsymbol{\varphi}}-2 \mu v_{\text {bin }} \hat{\boldsymbol{\varphi}}_{\text {bin }}}{e_{\text {out }}^{3} v_{K}^{3}}$.

\section{EVOLUTION OF BINARY ORBITAL ELEMENTS}

In paper I we studied the evolution of single planetesimals by following the primary orbital semi-major axis $a_{\text {out }}$, eccentricity $e_{\text {out }}$ and inclination $I_{\text {out }}$. For BPs, we will study the evolution of the inner binary separation $a_{b i n}$, binary eccentricity $e_{b i n}$ and binary inclination angle $I_{b i n}$, relative to the orbital plane of the primary around the star. In this section we derive the timescales for the change of binary separation and comment on changes in the binary eccentricity and inclination.

In its most general form, the differential acceleration in eqns. 12 and 17 can be decomposed into

$$
\Delta \boldsymbol{a}_{G D F}=\mathcal{A}_{\text {out }} \hat{\boldsymbol{\varphi}}+\mathcal{A}_{\text {bin }} \hat{\boldsymbol{\varphi}}_{\text {bin }},
$$

where $\mathcal{A}_{\text {out }}$ and $\mathcal{A}_{\text {bin }}$ depend on the specific regime and mass ratio. The total angular momentum of the binary per unit mass is $l=a_{b i n} v_{b i n}$, where $a_{b i n}$ is the binary separation. The change in the absolute angular momentum per unit mass is given by the specific torque, i.e. $\dot{l}=\left|\boldsymbol{a}_{\text {bin }} \times \boldsymbol{\Delta} \boldsymbol{a}_{G D F}\right|$. The average torque over one binary period is

$$
\left\langle\left|\boldsymbol{a}_{\text {bin }} \times \boldsymbol{\Delta} \boldsymbol{a}_{G D F}\right|\right\rangle=\frac{1}{2 \pi} \int_{0}^{2 \pi}\left|\boldsymbol{a}_{\boldsymbol{b i n}} \times \boldsymbol{\Delta} \boldsymbol{a}_{G D F}\right| d \nu_{b i n} .
$$

Let us consider a circular binary orbit, where $\hat{\boldsymbol{a}}_{\boldsymbol{b i n}} \perp$ $\hat{\boldsymbol{\varphi}}_{\text {bin }}$, and the averaged torque is

$$
\begin{aligned}
& \left\langle\left|\boldsymbol{a}_{\boldsymbol{b i n}} \times \boldsymbol{\Delta} \boldsymbol{a}_{G D F}\right|\right\rangle=\frac{a_{b i n}}{2 \pi} \int_{0}^{2 \pi}\left|\hat{\boldsymbol{\varphi}}_{\boldsymbol{b i n}} \cdot \boldsymbol{\Delta} \boldsymbol{a}_{G D F}\right| d \nu_{b i n} \\
& \left.=\frac{a_{b i n}}{2 \pi} \int_{0}^{2 \pi} \mid \mathcal{A}_{c m} \hat{\boldsymbol{\varphi}} \cdot \hat{\boldsymbol{\varphi}}_{\text {bin }}+\mathcal{A}_{b i n}\right) \mid d \nu_{b i n}=\mathcal{A}_{b i n} a_{b i n} .
\end{aligned}
$$

The last equality comes from the assumption on separation of times, given in Eqn. (9). In this approximation, $\left\langle\hat{\boldsymbol{\varphi}} \cdot \hat{\boldsymbol{\varphi}}_{\text {bin }}\right\rangle \approx 0$ since $\hat{\boldsymbol{\varphi}}_{\text {bin }}$ Oscillates very rapidly with respect to $\hat{\varphi}$, therefore $\hat{\varphi}$ is constant in the frame of the binary (similar to the approach used by Perets \& Murray-Clay 2011). The validity of the approximation depends on the binary separation (i.e. $\left\langle\hat{\boldsymbol{\varphi}} \cdot \hat{\boldsymbol{\varphi}}_{\text {bin }}\right\rangle=$ $\left.\left\langle\cos \left(\left(\Omega_{b i n}-\Omega\right) t\right)\right\rangle=f^{3 / 2}+\mathcal{O}\left(f^{3}\right)\right)$. It is roughly $<3 \%$ for $f=0.1$ but could be significant for larger separations. The inspiral timescale is then given by

$$
\tau_{\text {ins }}=\frac{1}{2} \frac{l}{\dot{l}}=\frac{v_{\text {bin }}}{\mathcal{A}_{\text {bin }}} .
$$

This is the timescale for which the binary loses approximately half of its initial separation, similarly to the definition of migration timescale in Paper I.

An alternative derivation of eqn (21) can be obtained from considering the equations of motion for a disturbing force. For the binary orbital elements, it is best to 
describe the change in orbital elements due to the disturbing force, and consequently, the disturbing acceleration $\boldsymbol{\Delta} \boldsymbol{A}=\Delta A_{r} \hat{\boldsymbol{r}}+\Delta A_{\varphi} \hat{\boldsymbol{\varphi}}+\Delta A_{z} \hat{\boldsymbol{z}}$ (Murray \& Dermott 1999), similarly to the case of single planetesimals in paper 1

$$
\begin{array}{r}
\frac{d a_{b i n}}{d t}=2 \frac{a_{b i n}^{3 / 2}}{\sqrt{G m_{b i n}\left(1-e_{b i n}^{2}\right)}} \\
\times\left[\Delta A_{r} e_{b i n} \sin \nu_{b i n}+\Delta A_{\varphi}\left(1+e_{b i n} \cos \nu_{b i n}\right)\right] \\
\frac{d e_{b i n}}{d t}=\sqrt{\frac{a_{b i n}\left(1-e_{b i n}^{2}\right)}{G m_{b i n}}} \\
\times\left[\Delta A_{r} \sin \nu_{b i n}+\Delta A_{\varphi}\left(\cos \nu_{b i n}+\cos E_{b i n}\right)\right] \\
\frac{d I_{b i n}}{d t}=\sqrt{\frac{a\left(1-e_{b i n}^{2}\right)}{G m_{b i n}}} \frac{\Delta A_{z} \cos \left(\omega_{b i n}+\nu_{b i n}\right)}{1+e_{b i n} \cos \nu_{b i n}},
\end{array}
$$

where $E_{b i n}$ is the eccentric anomaly and $\omega_{b i n}$ is the argument of peri-center of the binary. For circular binary, eqn 22 reduces to

$$
\frac{d a_{b i n}}{d t}=2 \frac{a_{b i n}^{3 / 2}}{\sqrt{G m_{b i n}}} \Delta A_{\varphi},
$$

and the inspiral time,

$$
\tau_{\text {ins }}=\frac{a_{b i n}}{\dot{a}_{b i n}}=a_{b i n} \sqrt{\frac{G m_{b i n}}{a_{b i n}^{3}}} \frac{1}{2 \Delta A_{\varphi}}=\frac{v_{b i n}}{2 \Delta A_{\varphi}},
$$

is the same as in eqn. 21), where $\Delta A_{\varphi}$ is recognized as $\mathcal{A}_{\text {bin }}$ when averaged over binary orbital period.

Given the latter, alternative derivation of eqn. (25), several points naturally rise. First, eqn $(22)$ is correct even if the binary has a small inner eccentricity, similarly to paper I. In addition, for binary with $f<0.1$, the terms proportional to trigonometric function of $\nu_{b i n}$ are averaged out to zero over one binary orbit, due to separation of times. Hence, $e_{b i n}$ and $I_{b i n}$ are constant at first approximation.

In the following we will calculate the inspiral timescale for each regime.

\subsection{Linear regime}

If the primary is in a nearly circular orbit, the Mach numbers involved in the GDF force are small, and we are able to apply the approximation of linear regime. In the linear regime, using Eqns. (21) and (21) the inspiral timescale is

$$
\tau_{\text {ins }}=\frac{1}{4 C_{\text {sub }} \mu}=\frac{3(1+q)}{16 \pi} \frac{c_{s}^{3}}{G^{2} \rho_{g} m_{s}} .
$$

For equal mass binaries with $m \sim 2 \cdot 10^{23} \mathrm{~g}$ we get $\tau_{\text {ins }} \sim$ $0.4 \mathrm{Myr}$. The inspiral timescale is comparable to the protoplanetary disk lifetime of $10 \mathrm{Myr}$ (Pfalzner et al.|2014) for planetesimals with mass of $m \sim 10^{22} \mathrm{~g}$. Thus, planetesimals with larger mass will inevitably merge, while planetesimals with lower mass can survive after the dispersal of the protoplanetary disk. Further in the disk, the gas density decreases as $\rho_{g} \propto a^{-16 / 7}$, thus the inspiral timescale is longer, and the critical mass for merger is larger. This fact has implications on the frequency and properties of BPs in the debris disk, and is discussed in the discussion.

It is worth noting that the merger timescale is obtained by integrating eqn. (21) to obtain $\tau_{\text {merge }}=$ $\tau_{\text {ins }} \ln \left(a_{i n} / R_{c}\right)$ where $a_{i n}$ is the initial separation and $R_{c}$ is the contact radius of the binary (Perets \& Murray-Clay 2011).

Note that the timescale in eqn. (21) is shorter than the migration timescale $\tau_{a}$ in paper I (eqn. 11) by a factor of $\approx H_{0}^{2}(1+q)$. It is due to the fact that for single planetesimals, the length scale involved is $a_{\text {out }} \sim v_{K} / \Omega \sim$ $H_{0}^{-2} v_{r e l} / \Omega$, while for BPs, it is $a_{b i n} \sim v_{b i n} / \Omega_{b i n}$. In the linear regime $(\mathcal{M} \ll 1)$ where $F_{G D F} \propto v_{\text {rel }}$, the characteristic time for the change in $a_{\text {out }}$, taking a relative velocity of $\sim v_{r e l}$ is $\sim H_{0}^{-2}$ longer than the characteristic time for the change in $a_{b i n}$ due to GDF with relative velocity $\sim v_{b i n}$.

It is worth noting that $\mathcal{A}_{\text {out }}=0$ for $q=1$. In addition, if $q \ll 1$, then $\mathcal{A}_{\text {out }} \gg \mathcal{A}_{\text {bin }}$, so that even if separation of times (Eqn. 9) is satisfied, the larger body inspirals to the center of mass much faster that its companion. Thus, for the remaining time of the evolution $v_{b}$, and consequently $\mathcal{A}_{\text {out }}$ vanish. Hence the inspiral time in eqn. (21) is valid.

\subsubsection{Deviations from the linear regime}

In paper I we have seen that for single planetesimals, the equations and times scale with the mass as $\tau \propto m_{p}^{-1}$. The latter is true for any Mach number, not only in the linear regime. One of the reasons is that the only relevant length scale is the semi-major axis $a_{\text {out }}$ (and consequently the only relevant velocity scale is $v_{K}$, which is unaffected by the mass of the planetesimal). For BPs this is not the case. An additional length scale, the binary separation $a_{b i n}$, exists (and consequently an additional velocity scale $v_{b i n}$ ), and it depends on the mass of the binary $m_{b i n}$. Changing the mass of the binary will result in a change in $v_{b i n}$, and consequently in the binary's Mach number and it's dynamical behavior due to GDF. However, for the linear regime, $\tau_{\text {ins }}$ given by eqn. 26 , will not depend neither on $a_{b i n}$, nor on $v_{b i n}$, and will scale as $\propto \mu^{-1}$.

We can calculate the deviation from the linear regime in terms of the Mach number, taking the next term in eqn. (4). The non-linear contribution is $\Delta a_{G D F}=$ $\Delta a_{G D F, l i n}\left(1+3 \mathcal{M}^{2} / 5\right)$ which leads to

$$
\tau_{\text {ins }}=\tau_{\text {ins }, \text { lin }}\left(1+\frac{3}{5} \mathcal{M}^{2}\right)^{-1} \approx \tau_{\text {ins }, \text { lin }}\left(1-\frac{3}{5} \mathcal{M}^{2}\right) .
$$

\subsection{Supersonic regime}

Consider now an equal mass binary on an eccentric orbit, such that $v_{c m}>c_{s}$ (or $\left.e_{\text {out }} \gtrsim 2 H_{0}\right)$. Generally, the Mach number changes with time. Let us decompose the Mach number into the orbital Mach number $\mathcal{M}_{c m} \equiv\left|\boldsymbol{v}_{\text {out }}\right| / c_{s}$, and the binary Mach number $\mathcal{M}_{\text {bin }} \equiv\left|\boldsymbol{v}_{\text {bin }}\right| / c_{s}$, similar to the approach of SánchezSalcedo \& Chametla (2014). We can use the impulse approximation, where the torque is maximal when the line connecting the binary components is parallel to the 


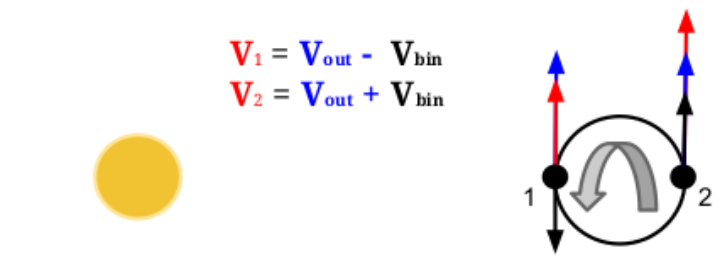

Subsonic: $\quad \mathbf{V}_{1}<\mathbf{V}_{2} \Rightarrow \mathbf{F} \propto-\mathbf{V} \quad$ and $\quad\left|\mathbf{F}_{1}\right|<\left|\mathbf{F}_{2}\right|$

Supersonic: $\mathbf{V}_{1}<\mathbf{V}_{2} \Rightarrow \mathbf{F} \propto-\mathbf{V}^{-2}$ and $\left|\mathbf{F}_{1}\right|>\left|\mathbf{F}_{2}\right|$

FIG. 1. - A sketch of a system containing the Sun and a binary planetesimal in orbit around it. Relative velocities are marked in red, the primary CM velocity in blue, and the binary velocity in black lines. Differential forces for the subsonic and the supersonic binaries are shown. Note that $v_{2}$ is always larger than $v_{1}$. For $\mathcal{M}<1$, the GDF force increases with velocity, and decreases with velocity for $\mathcal{M}>1$, hence the total torque is reversed (see details in text).

sun-CM line (i.e. $\nu_{\text {out }}=\nu_{\text {bin }}$ ) and where the binary Mach numbers, $\mathcal{M}_{\text {bin }}$ is maximized / minimized.

Fig. 1 illustrates the difference in the total torque between the subsonic and the supersonic binaries. For $\mathcal{M}_{1}<\mathcal{M}_{2}<1$, the induced torques are $T_{1}=a_{b i n} F_{1} / 2<$ $a_{b i n} F_{2} / 2=T_{2}$, and the total torque is positive. On the other hand, for $\mathcal{M}_{2}>\mathcal{M}_{1}>1$, the change in $\mathcal{I}(\mathcal{M})$ with respect to is $\mathcal{M}$ is negative, (i.e. $\operatorname{sgn}(d \mathcal{I}(\mathcal{M}) / d \mathcal{M})=$ -1 ), hence $T_{1}=a_{b i n} F_{1} / 2>a_{b i n} F_{2} / 2=T_{2}$ and the total torque is negative. As long as the supersonic orbit pertains, the torque is on the opposite direction and it adds angular momentum to the binary, and the binary gains orbital energy leading to increase in the binary separation.

The phenomena of increasing $a_{b i n}$ in the supersonic CM orbit can be connected to Fig. (12) of SánchezSalcedo \& Chametla (2014), where they calculate averaged radial and azimuthal forces for various values of $\mathcal{M}_{c m}$ and $\mathcal{M}_{\text {bin }}$. If both are subsonic, then $\left\langle F_{\varphi}\right\rangle$ is negative and Sánchez-Salcedo \& Chametla (2014) quote $\left\langle F_{\varphi}\right\rangle=-0.18$ (in normalized code units) for $\mathcal{M}_{c m}=0.5$ and $\mathcal{M}_{\text {bin }}=0.3$, but in our case $\mathcal{M}_{\text {bin }} \ll 1$, so $\left\langle F_{\varphi}\right\rangle$ is much lower. For $\mathcal{M}_{c m}=2.0$ and $\mathcal{M}_{\text {bin }}=0.5$, SánchezSalcedo \& Chametla (2014) quote that "the average of $\left\langle F_{\varphi}\right\rangle$ is close to zero". It is plausible that for our set of parameters (i.e. $\left.\mathcal{M}_{\text {bin }} \ll 1\right),\left\langle F_{\varphi}\right\rangle$ is positive. Since for the supersonic case $d I(\mathcal{M}) / d \mathcal{M}$ issteeper at the vicinity of the peak, we expect $\left|\boldsymbol{\Delta} \boldsymbol{a}_{G D F}\right|$ to grow as the orbit circularizes, and then abruptly to change sign. Then, for $\mathcal{M}_{c m} \lesssim 1+\mathcal{M}_{b i n}$ GDF becomes very efficient in extracting angular momentum, and the binary separation will sharply decrease.

Since the torque is reversed in the supersonic regime, we will define the "break-up timescale" similarly to the inspiral timescale as in eqn. 26). Using eqns. (17) and (21), the break-up timescale is

$$
\tau_{\text {break }}=\frac{e_{\text {out }}^{3} v_{K}^{3}}{4 \mu C_{\text {super }}}=\frac{e_{\text {out }}^{3} v_{K}^{3}(1+q)}{16 \pi m_{s} G^{2} \rho_{g} \ln \Lambda} .
$$

Comparison with the inspiral timescale in Eqn, 26. yields

$$
\frac{\tau_{\text {break }}}{\tau_{\text {ins }}}=\frac{1}{3 \ln \Lambda}\left(\frac{e_{\text {out }}}{H_{0}}\right)^{3}
$$

\begin{tabular}{|c|c|c|c|c|c|}
\hline \#run & $Q$ & $q$ & $e_{\text {out }}$ & $f$ & $e_{\text {bin }}$ \\
\hline \hline 1 & $2 \cdot 10^{-10}$ & 1 & 0 & 0.3 & 0 \\
\hline 2 & $2 \cdot 10^{-10}$ & 1 & 0 & 0.3 & 0.4 \\
\hline 3 & $2 \cdot 10^{-10}$ & 1 & 0 & 0.1 & 0 \\
\hline 4 & $2 \cdot 10^{-10}$ & 1 & 0 & 0.1 & 0.4 \\
\hline 5 & $2 \cdot 10^{-10}$ & 1 & 0.1 & 0.1 & 0 \\
\hline 6 & $2 \cdot 10^{-10}$ & 1 & 0.1 & 0.1 & 0.4 \\
\hline 7 & $2 \cdot 10^{-10}$ & 1 & 0.3 & 0.1 & 0 \\
\hline 8 & $2 \cdot 10^{-8}$ & 1 & 0 & 0.1 & 0 \\
\hline 9 & $2 \cdot 10^{-8}$ & 1 & 0 & 0.1 & 0.4 \\
\hline 10 & $2 \cdot 10^{-8}$ & 1 & 0.1 & 0.1 & 0 \\
\hline 11 & $2 \cdot 10^{-8}$ & 1 & 0.1 & 0.1 & 0.4 \\
\hline 12 & $10^{-8}$ & $10^{-2}$ & 0 & 0.1 & 0 \\
\hline 13 & $10^{-8}$ & $10^{-2}$ & 0.1 & 0.1 & 0 \\
\hline
\end{tabular}

TABLE 1

VARIOUS RUNS FOR DIFFERENT INITIAL CONDITIONS. THE NUMBERS ARE IN SIMULATION UNITS. IN ALL SIMULATIONS, THE SEMI-MAJOR AXIS OF THE ORBIT AROUND THE SUn, $a_{\text {out }}$, is 1.

and for $\ln \Lambda \simeq 10$ we get $\tau_{\text {break }} \approx 3.13 \cdot 10^{3} \tau_{\text {ins }} e_{\text {out }}^{3}$.

For larger eccentricities $e_{\text {out }} \gg H_{0}$, the break up timescale is long and the effect is negligible. The timescales are comparable for $e_{\text {out }} \approx 0.068$, and $\tau_{\text {break }} \approx$ $0.26 \tau_{\text {ins }}$ near the trans-sonic limit where $e_{\text {out }} \sim 2 H_{0}$. The binary break-up is determined by the initial separation. The decay of the outer eccentricity is $\dot{e}_{\text {out }} \sim-e_{\text {out }}^{-2}$ (Paper I), therefore the outer binary spends more time in higher eccentricities states. Note that the break up timescale is comparable to the eccentricity damping timescale (paper I), thus $e_{\text {out }}$ cannot be kept large enough to stay in the supersonic regime, and it is expected that for binaries with small enough separation, the binary expansion inevitably stops before the breakup. The final fate of the binary depends mostly on its initial separation. Determining the rate of supersonic expansion and the critical separation for break-up will be studied numerically and discussed in the discussion section. It is worth noting that for $q=1, \mathcal{A}_{\text {out }} \sim \mathcal{A}_{\text {bin }}$, and eqn. (21) is valid due to separation of times (Eqn. 9). The case of $q \ll 1$ is similar to the linear regime. Hence the expression for the inspiral time in eqn. (21) is also valid.

\section{NUMERICAL SET UP}

In order to model the detailed evolution of BPs numerically, we use GravityLab, an N-body integrator with a shared but variable time step, using the Hermite 4th order integration scheme documented in Hut et al. (1995)

The simulation units are $G=M_{\odot}=a_{\odot}=1$, hence at $1 \mathrm{AU}, v_{K}=1, T=2 \pi$. We add a fiducial GDF force that mimics the GDF force. At each step we calculate the additional external acceleration and jerk due to GDF. More Details of the modified code are summarized in the appendix of paper I. The disk properties are the same as in paper I and we neglect accretion and any other sources of drag other than GDF.

We run several N-body simulations with a central object and a bound binary planetesimal with various initial conditions. We first present the results for co-planar binaries $\left(I_{b i n}=0\right)$; which initial conditions are summarized in table 1. We then present the results for inclined binaries; which initial conditions are summarized in table 2. 

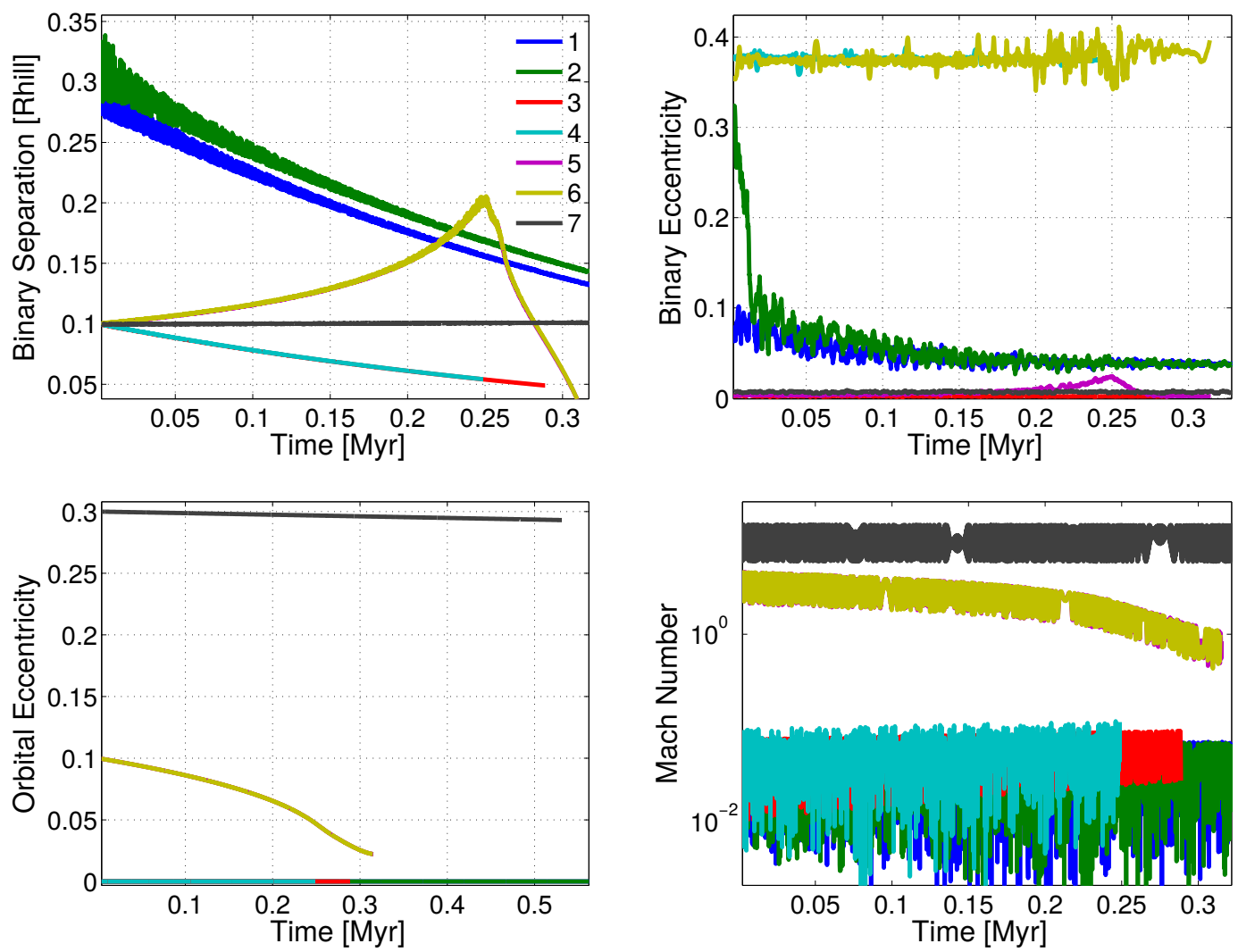

FIG. 2.- Evolution of co-planar binaries (runs 1-7). In each run the mass ratios are $Q=2 \cdot 10^{-10}$ and $q=1$. Top left: binary separation vs. time. Top right: binary eccentricity vs. time. For clarity, the data points are averaged such that each value is an average of 10 nearest data points, reducing the fluctuation noise. Bottom left: Orbital eccentricity vs. time. Bottom right: Mach number vs. time.

\begin{tabular}{|c|c|c|c|c|}
\hline \#run & $Q$ & $q$ & $e_{\text {out }}$ & $I_{\text {bin }}$ \\
\hline \hline 14 & $2 \cdot 10^{-8}$ & 1 & 0 & $0^{\circ}$ \\
\hline 15 & $2 \cdot 10^{-8}$ & 1 & 0 & $30^{\circ}$ \\
\hline 16 & $2 \cdot 10^{-8}$ & 1 & 0 & $60^{\circ}$ \\
\hline 17 & $2 \cdot 10^{-8}$ & 1 & 0 & $120^{\circ}$ \\
\hline 18 & $2 \cdot 10^{-8}$ & 1 & 0 & $150^{\circ}$ \\
\hline 19 & $2 \cdot 10^{-8}$ & 1 & 0 & $180^{\circ}$ \\
\hline 20 & $2 \cdot 10^{-8}$ & 1 & 0.1 & $0^{\circ}$ \\
\hline 21 & $2 \cdot 10^{-8}$ & 1 & 0.1 & $30^{\circ}$ \\
\hline 22 & $2 \cdot 10^{-8}$ & 1 & 0.1 & $60^{\circ}$ \\
\hline 23 & $2 \cdot 10^{-8}$ & 1 & 0.1 & $120^{\circ}$ \\
\hline 24 & $2 \cdot 10^{-8}$ & 1 & 0.1 & $150^{\circ}$ \\
\hline 25 & $2 \cdot 10^{-8}$ & 1 & 0.1 & $180^{\circ}$ \\
\hline 26 & $10^{-8}$ & $10^{-2}$ & 0 & $0^{\circ}$ \\
\hline 27 & $10^{-8}$ & $10^{-2}$ & 0 & $60^{\circ}$ \\
\hline 28 & $10^{-8}$ & $10^{-2}$ & 0 & $120^{\circ}$ \\
\hline 29 & $10^{-8}$ & $10^{-2}$ & 0.1 & $0^{\circ}$ \\
\hline 30 & $10^{-8}$ & $10^{-2}$ & 0.1 & $60^{\circ}$ \\
\hline 31 & $10^{-8}$ & $10^{-2}$ & 0.1 & $120^{\circ}$ \\
\hline
\end{tabular}

TABLE 2

VARIOUS RUNS FOR DIFFERENT INITIAL CONDITIONS. THE NUMBERS ARE IN Simulation Units. MACH NUMBER IS NOT AN INITIAL CONDITION, BUT RATHER DERIVED FROM THE RELATIVE VELOCITY OF THE INITIAL ORBIT. IN ALL SIMULATIONS, THE SEMI-MAJOR AXIS TO THE CENTER OF MASS OF THE BINARY Planetesimal, $a_{\text {out }}$ IS 1.

\section{RESULTS}

In the following we present the results of the evolution of BPs due to GDF. We first explore the parameter space of co-planar BPs (Table 11) and then we present the results of inclined BPs (Table2).
In order to check eqns. 21 and 28, we ran several simulations with different values of $c_{s}$ and found that $\tau_{\text {ins }} \propto c_{s}^{3}$ and that $\tau_{\text {break }}$ is independent of $c_{s}$, as expected.

\subsection{Co-planar Binaries}

7.1.1. Equal mass binaries, $q=1$

In figure (2), we plot the evolution of BPs from runs 17. On the top left panel, we plot the evolution of binary separation versus time. Runs 1 and 2 had started with $f=0.3$ while runs 3 and 4 had started with $f=0.1$. We see that they both inspiral at the same timescales, hence $\tau_{\text {ins }}$ does not depend on the binary separation $f$. Runs 5 and 6 had started with $e_{\text {out }}=0.1$ and indeed, the binary is seen to gain angular momentum and expand as long as the regime is supersonic, and the expansion rate increases as the Mach number decreases, as expected. The binary eccentricity $e_{b i n}$ is not important, and the plots of runs 5 and 6 are indistinguishable. Run 7 had started with $e_{\text {out }}=0.3$. Due to the large Mach numbers involved, GDF has little effect on the binary. On the top right panel we plot the evolution of the binary eccentricity versus time. We see that for runs with $f=0.3, e_{\text {bin }}$ drops rapidly, and stays constant for $f=0.1$. The reason is separation of times (9), and the RHS terms in eqn. 23 oscillates rapidly, such that $\left\langle\dot{e}_{b i n}\right\rangle_{n} \approx 0$, while for $f=0.3$ the latter is not true. Note that in cases where the separation of runs 5 and 6 is maximal $\left(a_{b i n} \sim 0.2 r_{H}\right)$, the amplitude of the oscillations of $e_{b i n}$ are larger for the same reason. In order to test the dependence on 

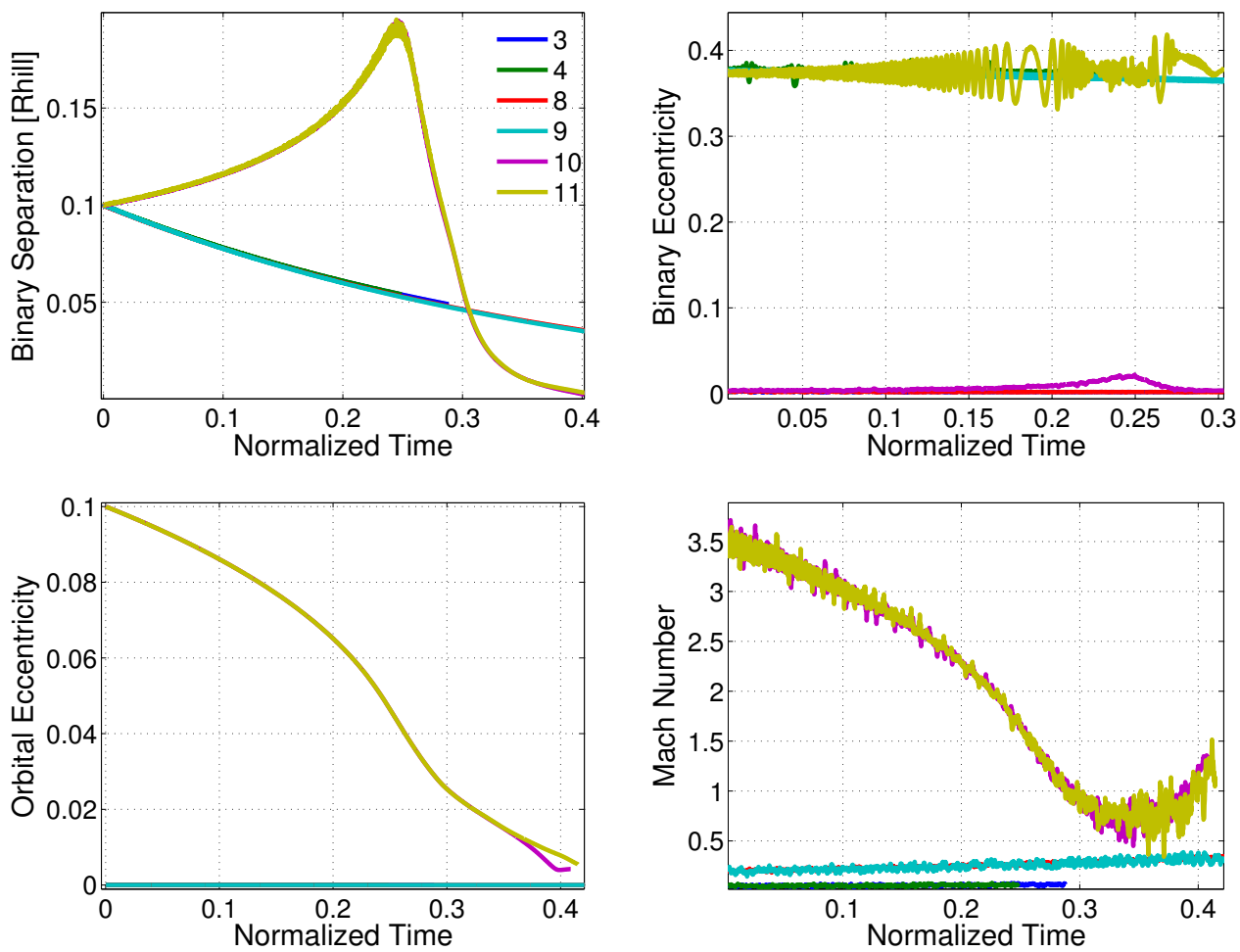

Fig. 3.- Evolution of co-planar binaries (runs 3-4 and 8-11). The initial mass ratios are $Q=2 \cdot 10^{-10}$ and $Q=2 \cdot 10^{-8}$ respectively, and $q=1$. For runs with $Q=2 \cdot 10^{-8}$ we multiplied the time by 100 to check the deviation from the linear regime. Runs with $Q=2 \cdot 10^{-10}$ are displayed in their real time (in Myr). Top left: binary separation vs. time. Top right: binary eccentricity vs. time. Bottom left: Orbital eccentricity vs. time. Bottom right: Mach number vs. time. Plotted data points are averaged, as in Fig. 2

eccentricity $\tau_{\text {break }} \propto e_{\text {out }}^{3}$ from Eqn 28 , we have used a power law fit to the data in run5 and got a power law fit of $\tau_{\text {break }} \propto e_{\text {out }}^{2.993}$, consistent with the expectations. In the bottom left panel we plot the orbital eccentricity versus time. The eccentricity damping has the same trend for $e_{\text {out }}=0.1$, while only a little effect is observed for for $e_{p}=0.3$. Finally, at the bottom right we plot the Mach numbers of one of the binary members versus time. We see that the transition to the subsonic regime for runs 5 and 6 is consistent with the eccentricity damping in the previous panel.

In figure (3), we plot the evolution of BPs from runs $3-4$ and $8-11$. For runs with $Q=2 \cdot 10^{-8}$ the time has been re-normalized (see fig. description). The panels are the same as in fig. 2. In the top left panel, the trend is the same as in fig. 2. The same trend is also evident in the eccentricities on the top right and bottom left panels. The only difference is seen in the top right panel, where the Mach numbers of the more massive binaries (runs 8 and 9) are larger than in the cases of the less massive binaries (runs 3 and 4 ). The latter is due to the fact that the binary velocity scales as $v_{\text {bin }} \propto Q^{1 / 3}$. For runs 10 and 11, the Mach numbers decrease as $e_{\text {out }}$ decreases, and increase again when the binary rapidly inspirals and $v_{b i n}$ increases to sonic velocities before the binary merger.

\subsubsection{Deviation from linear regime}

In fig. 3, the most visible difference between runs 3 and 8 (that have identical initial conditions except for $Q)$ is the Mach number. We can estimate the deviation from the linear regime by studying the small differences in the binary separation. We examine the bi- nary separation of runs 3 and 8 at $t_{f}=2.5$ normalized time units, where both runs have lost $\sim 1 / 2$ their initial separation. At $t_{f}, f_{3}=0.054$ for run 3 , and $f_{8}=0.053$ for run 8 . The difference between both runs is $\Delta f / f \approx 1.85 \%$. For run 8 , the Mach number at $t_{f}$, the Mach number is $\mathcal{M}_{3}=0.09$ for run 3 and $\mathcal{M}_{8}=0.267$, which leads to relative error of eqn. 27 of $\delta \tau_{\text {ins }} / t_{f}=\left(1-\left(1-0.6 \mathcal{M}_{8}^{2}\right) /\left(1-0.6 \mathcal{M}_{3}^{2}\right)\right) \approx 3.8 \%$. We conclude that the deviation is within the estimated error in eqn. 27 .

\subsubsection{Low mass ratio binaries $(q \ll 1)$}

In fig. 4 we compare runs 12 and 13 with $q=10^{-2}$ with previous runs 8 and 10, for which the initial conditions are the same except for $q$. For hierarchical binaries with $q \ll 1$, the general trend is that the orbital primary elements (with subscript "out") scale with the binary to star mass ratio $Q$, and the binary elements scale with the reduced mass $\mu$ ( for small $q, \mu$ is the smaller mass). In the top left panel, we multiply the time by 50 to check the consistency of the linear regime. Binaries 8 and 12 inspiral in the same phase. Due to the small $q$ of run 13, its supersonic phase is short, and later on it inspirals similarly to previous runs. In the top right panel, the orbital eccentricities of runs 10 and 13 decay in the same fashion, but run 10 inspirals fast, the Mach number is close to the sonic limit, and the eccentricity damping is more efficient, while for run 13 the Mach numbers involved are subsonic. In the bottom left panel, we multiplied the time by two for runs with $Q=10^{-8}$ to account for the difference in $Q$. Runs with $Q=2 \cdot 10^{-8}$ are presented with their real time (in Kyrs). 

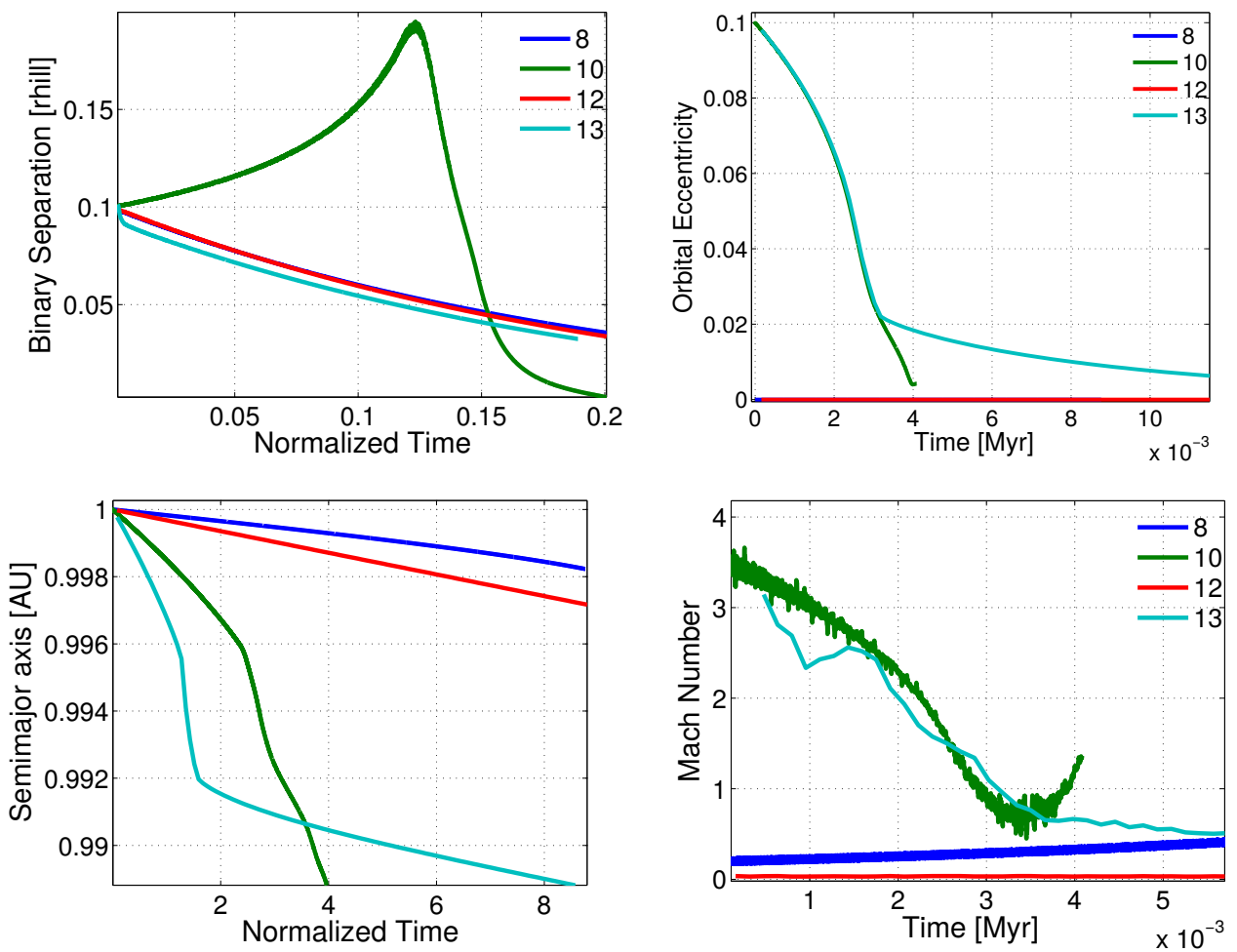

FIG. 4. - Evolution of co-planar binaries (runs 8,10 and 12,13). The initial mass ratios are $Q=2 \cdot 10^{-10}$ and $Q=10^{-8}$ respectively, and $q=1$ and $q=10^{-2}$ respectively. Top left: binary separation vs. time. For runs with $q=1$ we multiplied the time by 50 to compare different mass ratios. Runs with $q=10^{-2}$ are displayed in their real time (in Myr). Top right: binary eccentricity vs. time. Bottom left: Semi-major axis versus time. The time is multiplied by 2 for runs 12 and 13 since their $Q$ is smaller by a factor of two. Bottom right: Mach number over time. Plotted data points are averaged, as in Fig. 2 (but using only 5 nearest ata points).

In addition, note runs 3 and 12 in figures (2) and (4). They have the same parameters for the inspiral time, except $q$, and indeed, the inspiral time of run 12 is twice as fast.

The inward migration of the primary in runs with $q \ll 1$ is effectively similar to migration of single planetesimals. These planetesimals migrate faster than their equal mass binary counterparts. This is due to loss of gravitational focusing, discussed in Sánchez-Salcedo \& Chametla (2014) and Baruteau et al. (2011). The latter is true as long as the other parameters are comparable. At some point, the relevant Mach number for run 10 is $\mathcal{M}_{\text {bin }}$, and when it becomes supersonic, it dominates also the evolution of the primary orbital elements. As a consequence, the $\mathrm{CM}$ in run 10 continues to migrate inward at the same phase, while the CM migration in run 13 is slowed down due to the circularization of the primary and the low Mach numbers of the binary revolution. On the top right panel, the picture is similar to Fig. 3, where the more massive binaries involve larger Mach numbers.

To conclude this section, there is a good consistency between the predicted inspiral timescales and the simulations. The results are consistent with different mass ranges $Q$ and $q$, and deviation from the linear regime is well captured by eqn. 27. The supersonic expansion rate is also consistent with the prediction of eqn. 28.

\subsection{Inclined binaries}

\subsubsection{Equal mass binaries $(q=1)$}

On the left panel of fig. (5) we plot the evolution of binary elements of equal mass of $Q=2 \cdot 10^{-8}$ circular
CM orbit.

On the top left panel we see that the binary inspiral rate is indifferent to binary inclination. The binary inspiral rate deviates from that of co-planar binaries due to the increased periods of time that the binary spends at higher eccentricities due to the effects of secular evolution through Kozai-Lidov (KL) cycles Kozai (1962); Lidov (1962), which can significantly affect BPs (Perets \& Naoz 2009). For higher eccentricity, the Mach numbers involved are higher and GDF is more efficient. Since the KL timescale scales as $T_{K L} \propto a_{b i n}^{-3 / 2}$, the time spent at higher eccentricities increases with cycle number. The top right and bottom left panels show the evolution of binary eccentricity and inclination, respectively. Again, the KL timescales increase due to binary inspiral. On the bottom right panel we plot the evolution of the Mach numbers. Generally, a highly inclined binary contributes less to the relative velocity, and the average Mach number is lower than the co-planar orbit case. The higher Mach numbers after $\sim 7 \mathrm{Kyr}$ are due to the fact that the inclined binary is inspiraling faster than the circular binary near the sonic limit where the Mach numbers involved are close to unity.

On the left panels of fig. (6) we plot the binary eccentricity (top) and inclination (bottom) where we zoom-in on the first $600 \mathrm{yr}$. The KL timescale is given by $T_{K L} \approx P_{\text {out }}^{2}\left(1-e_{\text {out }}^{2}\right)^{3 / 2} / P_{\text {bin }}$, where $P_{\text {out }}=1 y r$ is the period of the primary, and $P_{\text {bin }}=f^{3 / 2} P_{\text {out }}$ is the binary period. For $e_{\text {out }}=0$, the KL timescale is $T_{K L}=f^{-3 / 2} y r$. The initial timescale is $T_{K L} \approx 32 \mathrm{yr}$ for 

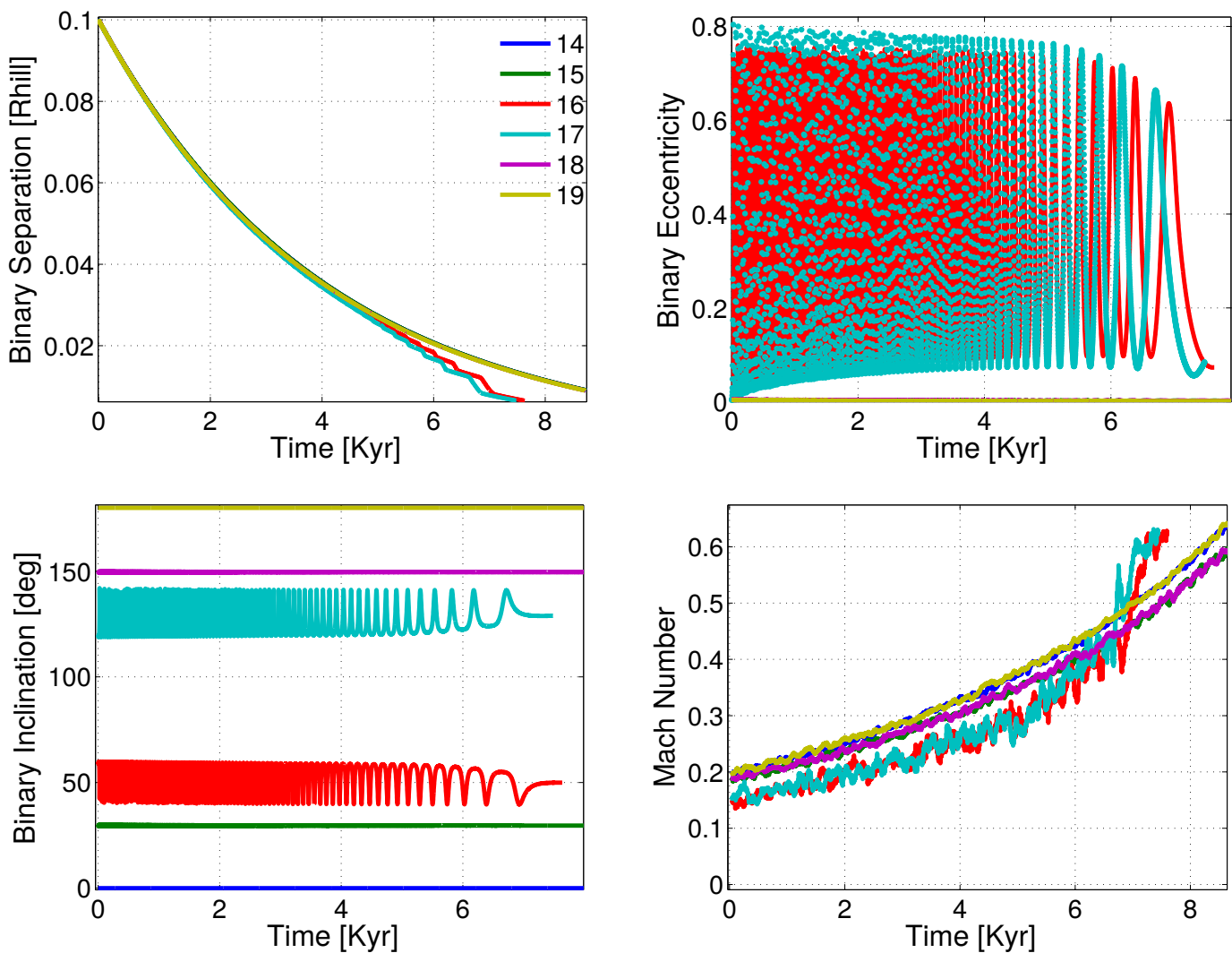

FIG. 5.- Binary elements for $Q=2 \cdot 10^{-8}$ and equal mass binaries with with various binary inclinations. Top left: Evolution of the binary separation. Top right: Evolution of the binary eccentricity. To avoid overlapping, the data of run 17 is plotted as scatter. Bottom left: Evolution of the binary inclination. Bottom right: Evolution of the Mach number. The data for Mach number have been averaged with 50 nearest data points to reduce fluctuations.
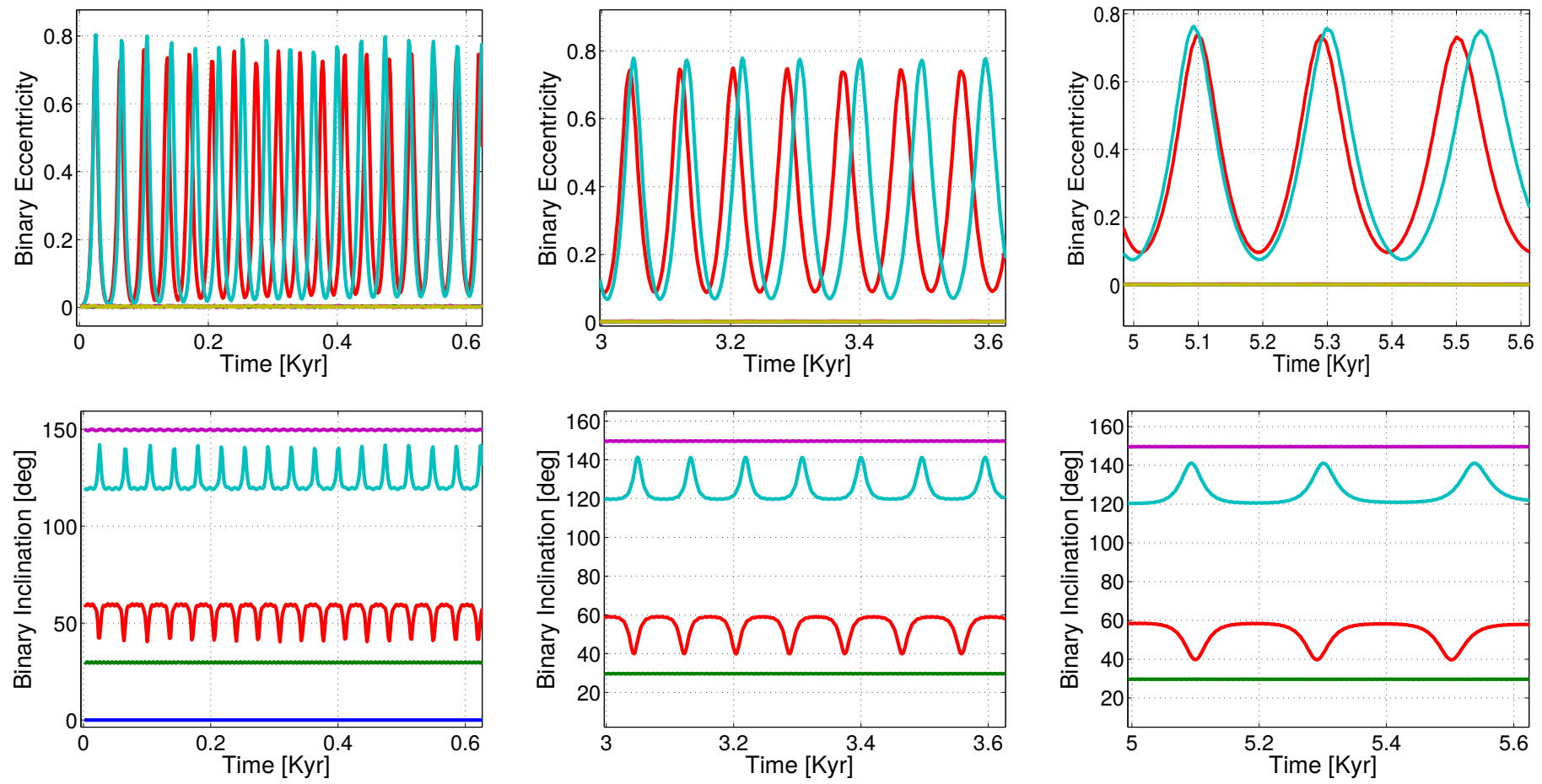

FIG. 6. - Zoom-in on the binary eccentricity (top) and inclination (left) evolution. The zoomed-in regions span $\sim 0.6 \mathrm{Kyrs}$ with starting time of 0,3 and 5 Kyrs, from left to right, respectively. 

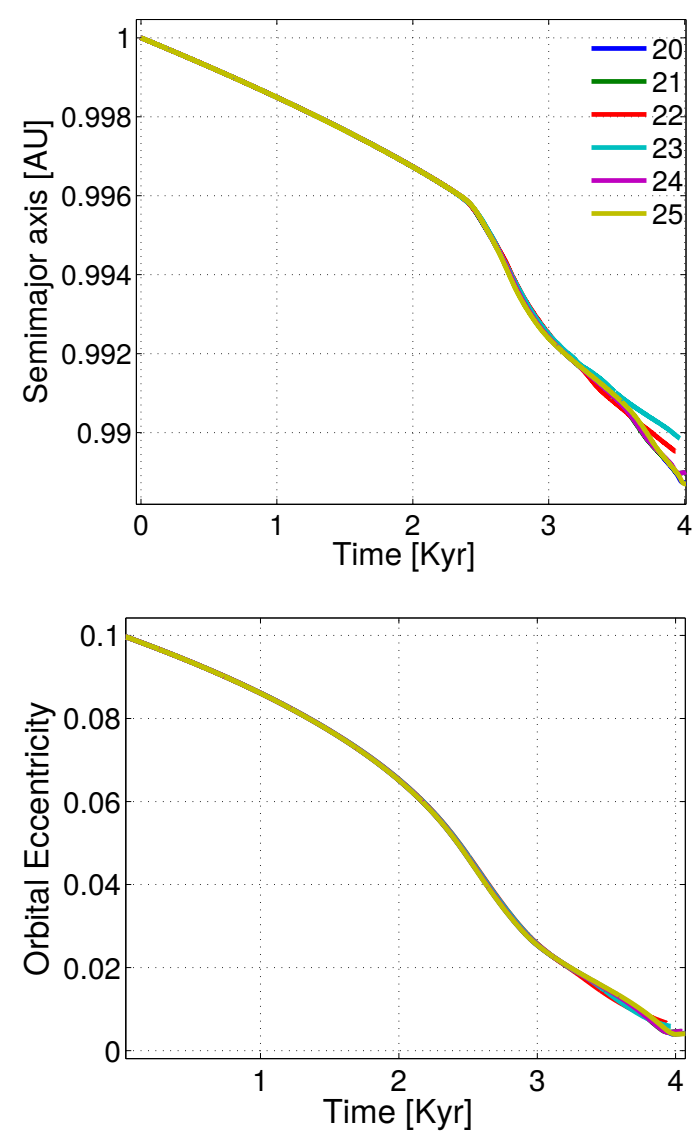

Fig. 7.- Evolution of the orbital semi-major axis (left) and eccentricity (right) for runs 20-25.

$f=0.1$, where $T_{K L}$ increases as the binary inspiral. At $t=3 K y r, f=0.05$ and $T_{K L} \approx 90 \mathrm{yr}$ (middle panels), and at $t=5 \mathrm{Kyr}, f \approx 0.027$ and $T_{K L} \approx 230 \mathrm{yr}$ (right panels).

We now turn to investigating inclined binaries with eccentric primary orbit (initial eccentricity $e_{\text {out }}=0.1$ ). In Fig. 7 we plot the orbital semi-major axis (left) and the eccentricity (right). We see that the decay of $a_{\text {out }}$ and $e_{\text {out }}$ is insensitive to the binary orbital elements, as expected. The orbital semi-major axis $a_{\text {out }}$ and eccentricity $e_{\text {out }}$ are insensitive to the inner structure of the binary. The latter breaks down only when $\mathcal{M}_{\text {bin }} \gtrsim \mathcal{M}_{c m}$.

In Fig. 8 we plot the binary orbital elements. On the top left panel we see the general feature of the supersonic expansion and rapid inspiral. The difference is that for larger inclinations the expansion rate is quenched, and for inclinations of 60 and $120 \mathrm{deg}$ there is no expansion. The reason is that the gained angular momentum is now used to damp the binary inclination (bottom left panel). In the bottom right panel, we see that the Mach numbers are dominated by $e_{\text {out }}$ for the first $3 \mathrm{Kyr}$, where $e_{p} \gtrsim$ $2 H_{0}$. Later on, the Mach numbers are dominated by the binary inspiral, where the differences between binary separations is small.

In Fig. 9 we plot the zoomed-in snapshots of the evolution of the binary eccentricity (top) and the inclination (bottom). Contrary to Fig. 6, the KL timescale $T_{K L}$ does not vary much, since $a_{b i n}$ is roughly constant. Only around $\sim 2.8 \mathrm{Kyr}$ does the binary begin to inspiral rapidly and the KL timescale increases. The binary eccentricity initially oscillates in the range of $\sim 0-0.8$, and in range of $\sim 0.3-0.5$ for prograde orbit and $\sim 0.4-0.65$ for retrograde orbit. It hints that the retrograde orbits are excited to higher eccentricities and are less stable for some configurations, as will be shown below. The inclinations are reduced by $\sim 30 \%$ near $t \sim 2.8 \mathrm{Myr}$.

\subsubsection{Low mass ratio binaries $(q \ll 1)$}

We now turn to binaries with small mass ratio $q \ll 1$.

In Fig. 10 we plot the evolution of hierarchical binaries with either circular $\left(e_{\text {out }}=0\right)$ or eccentric $\left(e_{\text {out }}=0.1\right)$ outer orbits. The primary orbital elements $\left(a_{p}, e_{p}\right)$ evolve similarly to the co-planar case (runs 12 and 13, Fig 4). On the top left panel we plot the evolution of the binary separation versus time. Due to the small mass ratio $q \ll 1$, the maximal change in separation for the supersonic regime is shorter by a factor of $q$, since the supersonic regime timescale is dominated by the outer eccentricity decay, $\dot{e}_{\text {out }}$, which is proportional to $m_{b}$. Hence, the supersonic regime timescale is shorter. For most of the orbits, the binary inclination and eccentricity follow the KL evolution. The exception is the retrograde orbit of run 31, which evolution is erratic. As the orbital eccentricity decreases and the Mach number approaches the sonic limit from above $\left(\mathcal{M} \rightarrow 1^{+}\right)$, the binary is subjected to large jerks due to the steep slope of $d \mathcal{I}(\mathcal{M}) / d \mathcal{M}$. The differential GDF force excites the binary eccentricities and the (sine of the) inclinations to larger values approaching unity. The inclination flips to prograde at $t \approx 2.7 \mathrm{Kyr}$. The orbit stays prograde for $\approx 200 \mathrm{yr}$ and then it goes back to retrograde. Later on the evolution returns to regular KL evolution with slightly higher inclination.

The dramatic GDF-induced dynamical effects bear no signature on the Mach number (bottom right) and the orbital evolution of the primary.

The combination of high inclination, small $q$ and large GDF jerks near the sonic limit $\mathcal{M} \gtrsim 1$ could lead to erratic behavior of the binary. In principle, the interaction of the lower mass body with the gas and the other two bodies depends on the binary phase. Although the long term evolution does not depend on the phase, the binary phase can be a crucial parameter when short term transient interactions are taking place, and large instantaneous GDF jerks are involved. In order to demonstrate that, we simulate the evolution of binary inclinations and eccentricities of five binaries with random initial phases.

Fig. 11 shows the evolution of binary inclinations and eccentricities for initial inclinations of $60^{\circ}$ (top panel) and $120^{\circ}$ (bottom panel). Similarly, Fig. 12 shows the evolution of binary inclinations and eccentricities for initial inclinations of $70^{\circ}$ (top panel) and $110^{\circ}$ (bottom panel). In both figures prograde orbits are more stable; they all survive and end up with somewhat higher inclinations and eccentricities. For the orbits with the largest inclinations attained, post-interaction oscillations on longer, secular timescales are evident, that decay over time. Retrograde orbits are prone to "torque kicks" that change the energy of the binary. Retrograde orbits are far less stable. Three of the five orbits for $120^{\circ}$ and two of the five orbits for $110^{\circ}$ have been destroyed. The rest have flipped their orientation at least once. Some of them remained prograde. The maximal eccentricities attained 

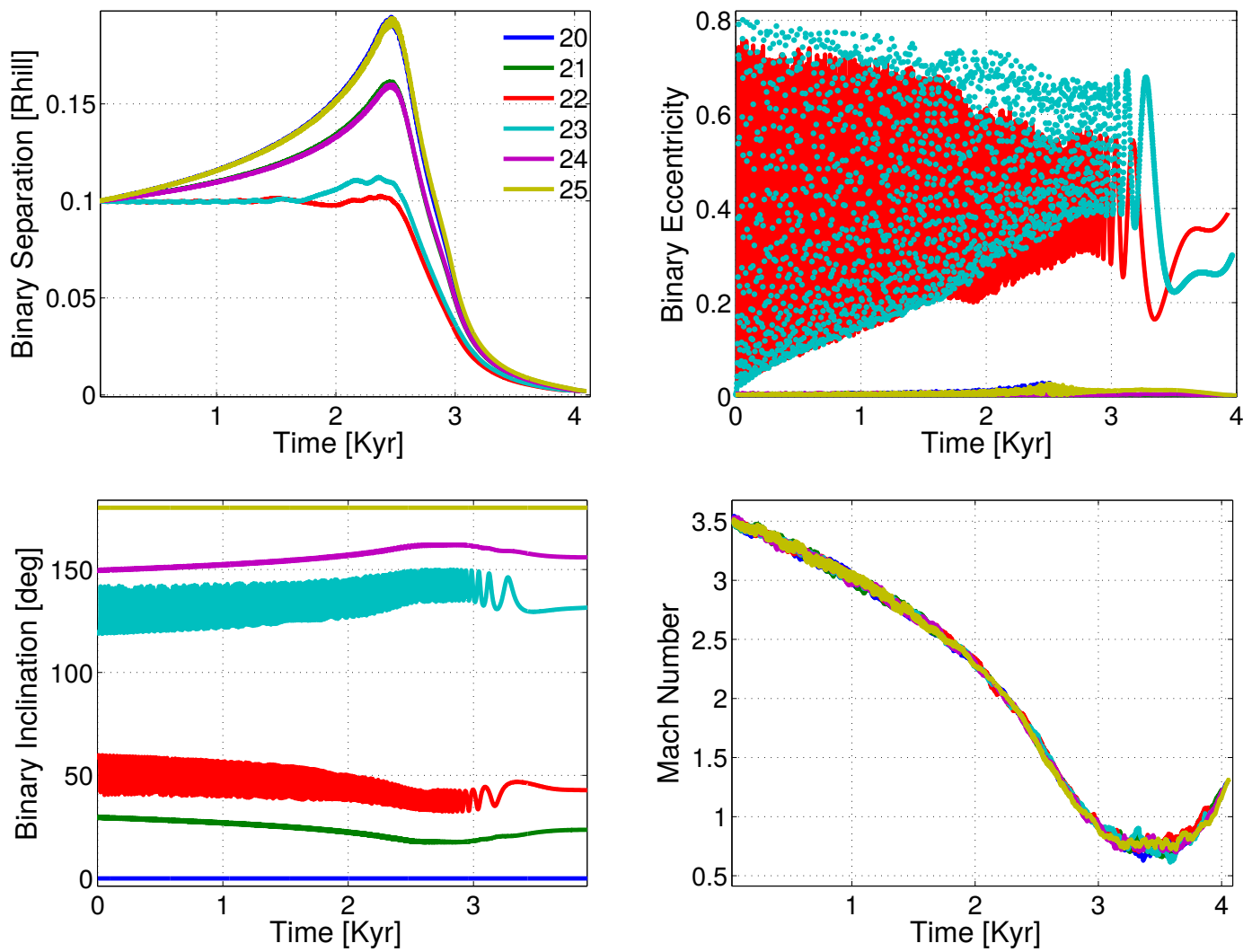

FIG. 8. - Same as Fig. 5 but with orbital eccentricity $e_{\text {out }}=0.1$
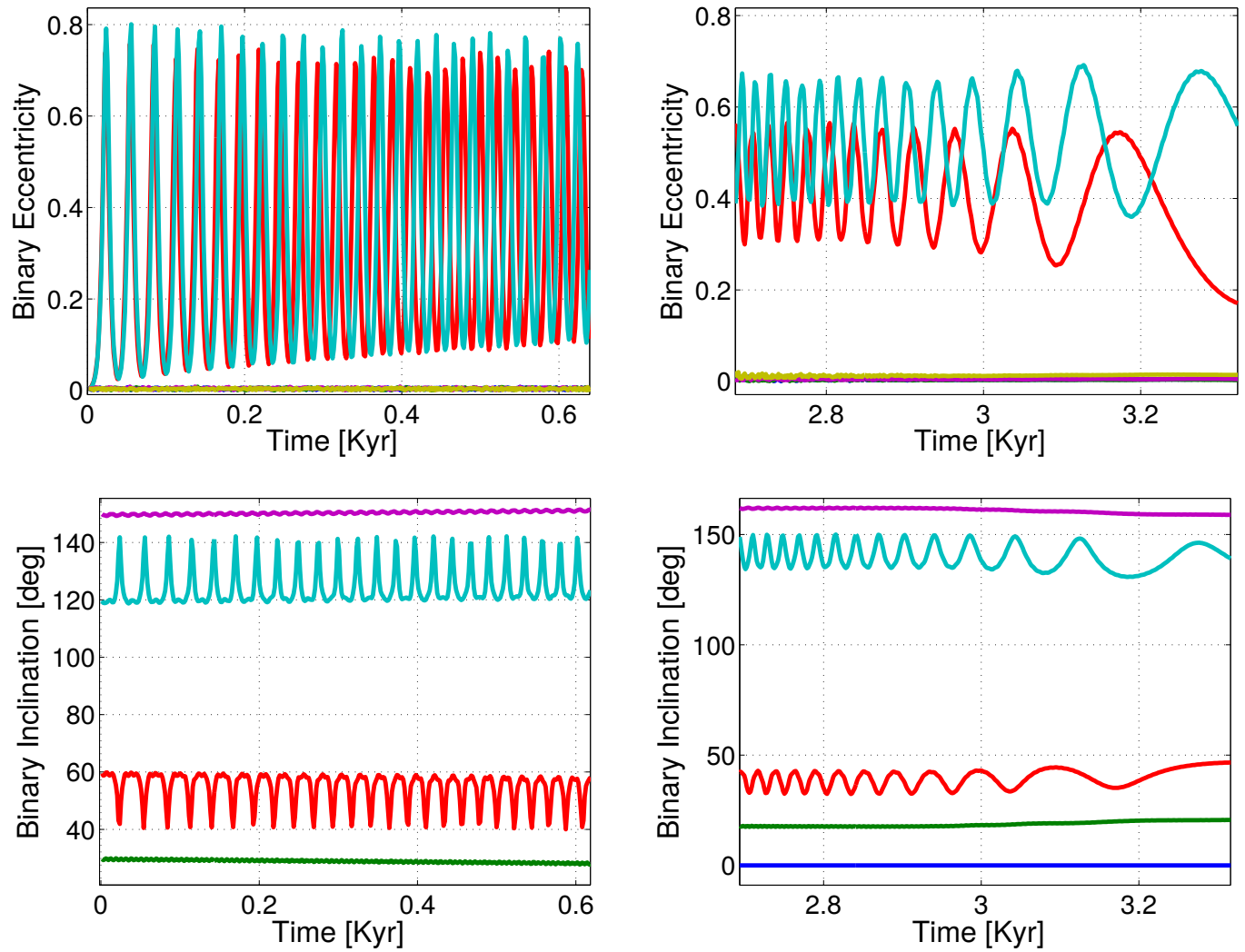

FIG. 9.- Same as figs (6) but with $e_{\text {out }}=0.1$ 

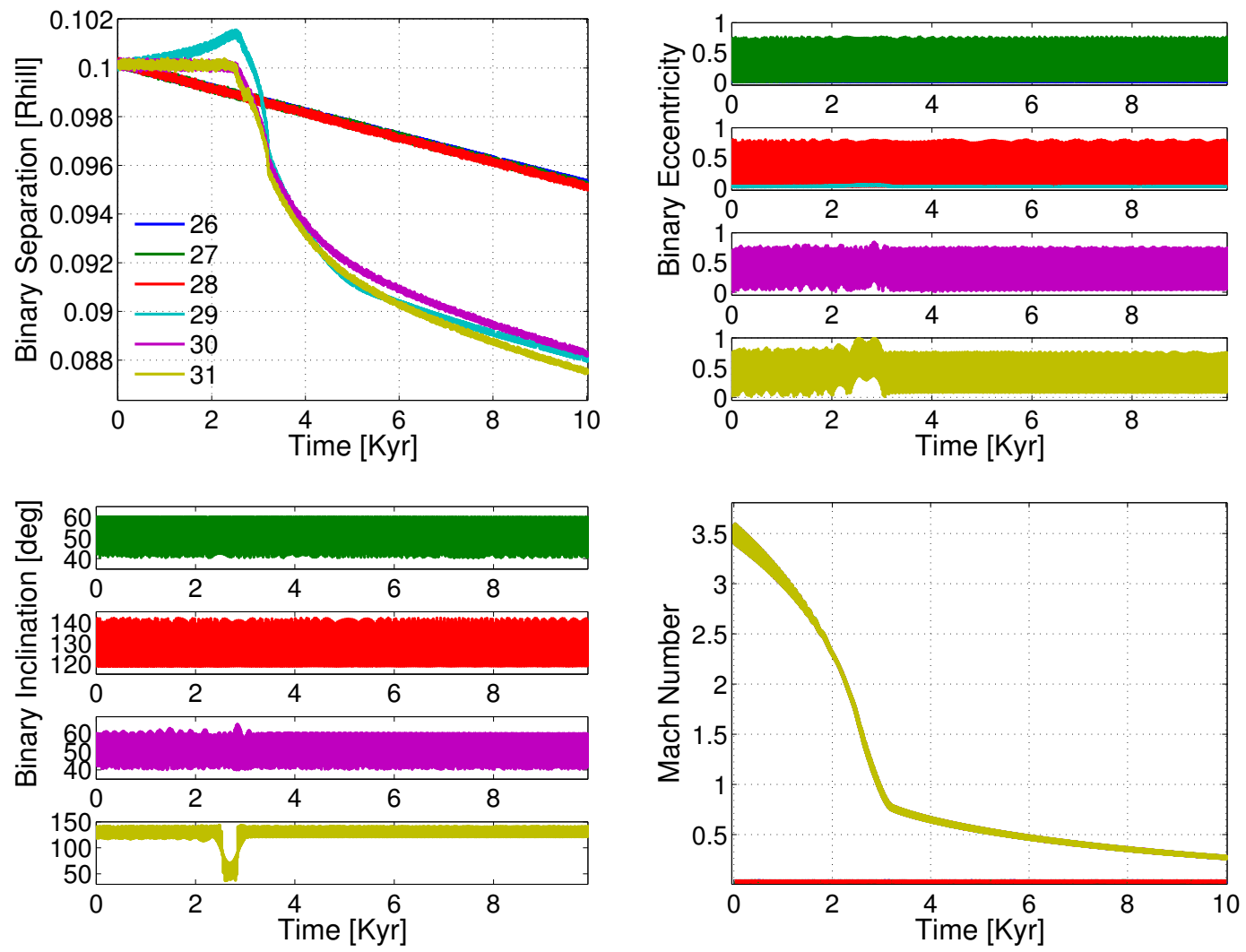

Fig. 10. Evolution of hierarchical binaries with $Q=10^{-8}$ and $q=10^{-2}$ with various binary inclinations and CM outer eccentricity (see Table 2 for list of initial conditions). Top left: Evolution of the binary separation. Top right: Evolution of the binary eccentricity. Bottom left: Evolution of the binary inclination. Bottom right: Evolution of the Mach number. 

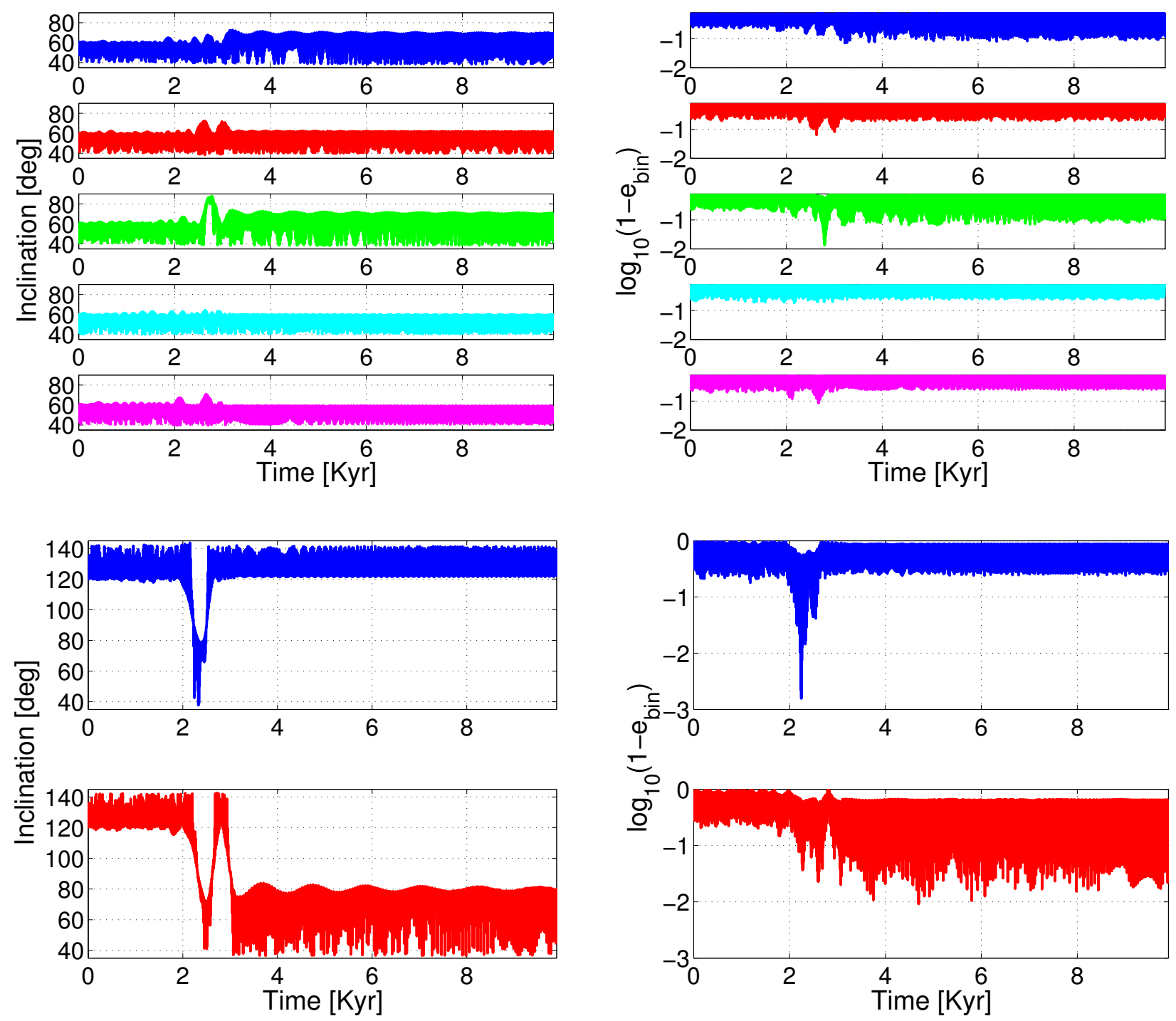

FIG. 11. - Evolution of binary orbital elements with random phase (i.e. true anomaly $\nu_{\text {bin }}$ is chosen randomly), $q=10^{-2}, Q=10^{-8}$, and outer eccentricity $e_{o u t}=0.1$. Top panels: $I=60 \mathrm{deg}$. Bottom panels: $I=120 \mathrm{deg}$. Left: Binary inclinations. Right: Binary eccentricity. $2 / 5$ of the retrograde binaries have survived and are presented here.

are in the range of $\log _{10}\left(1-e_{\text {bin }}\right) \sim(-2)-(-3)$. Given the above results, retrograde orbits are less stable and prone to spin flips and destruction of the BPs.

To conclude this section, the dynamical behavior of the primary orbital elements for inclined binaries is the same as for co-planar binaries. For low inclinations, the evolution of the binary elements is also similar. For higher inclinations, the binaries are subjected to rapid KL oscillations. If the outer eccentricity $e_{\text {out }}=0$, then the behavior of the binary orbital elements is similar to the co-planar case. If on the other hand, the initial eccentricity is $e_{\text {out }}=0.1$ (or any other large enough value), then the highly inclined orbits quench the inspiral, and the loss (or gain) of angular momentum leads to changes in $I_{b i n}$ and $e_{b i n}$. For low mass binary ratio $q \ll 1$, and particular binary orientation, the binary is excited to high inclinations and eccentricities near the trans-sonic limit $\mathcal{M} \gtrsim 1$. Prograde BPs survive and remain close to their initial conditions and evolve regularly with KL cycles. Retrograde BPs flip their angular momentum vector, and some of them collide and/or destroyed.

\section{DISCUSSION}

We have explored the evolution of intermediate mass BPs due to GDF by mimicking the effects of the gaseous disk as a fiducial external force in N-body simulations. The GDF force was derived following Ostriker s analytical theory. Naturally, it has limitations and does not take into account other physical processes (e.g. companion wake, non-linear effects, an inhomogenous medium, disk instabilities, turbulence etc.). Further studies of the evolution of a bound binary embedded in gaseous halo are required to model these additional effects. Detailed hydrodynamical simulations could lead to a better understanding of these processes and obtain the merger or break up timescale more rigorously.

The dynamical evolution of BPs can have important consequences for planet formation and for the current structure of single merger-formed planetesimals (asteroids/Kuiper-belt objects) and the configuration of 

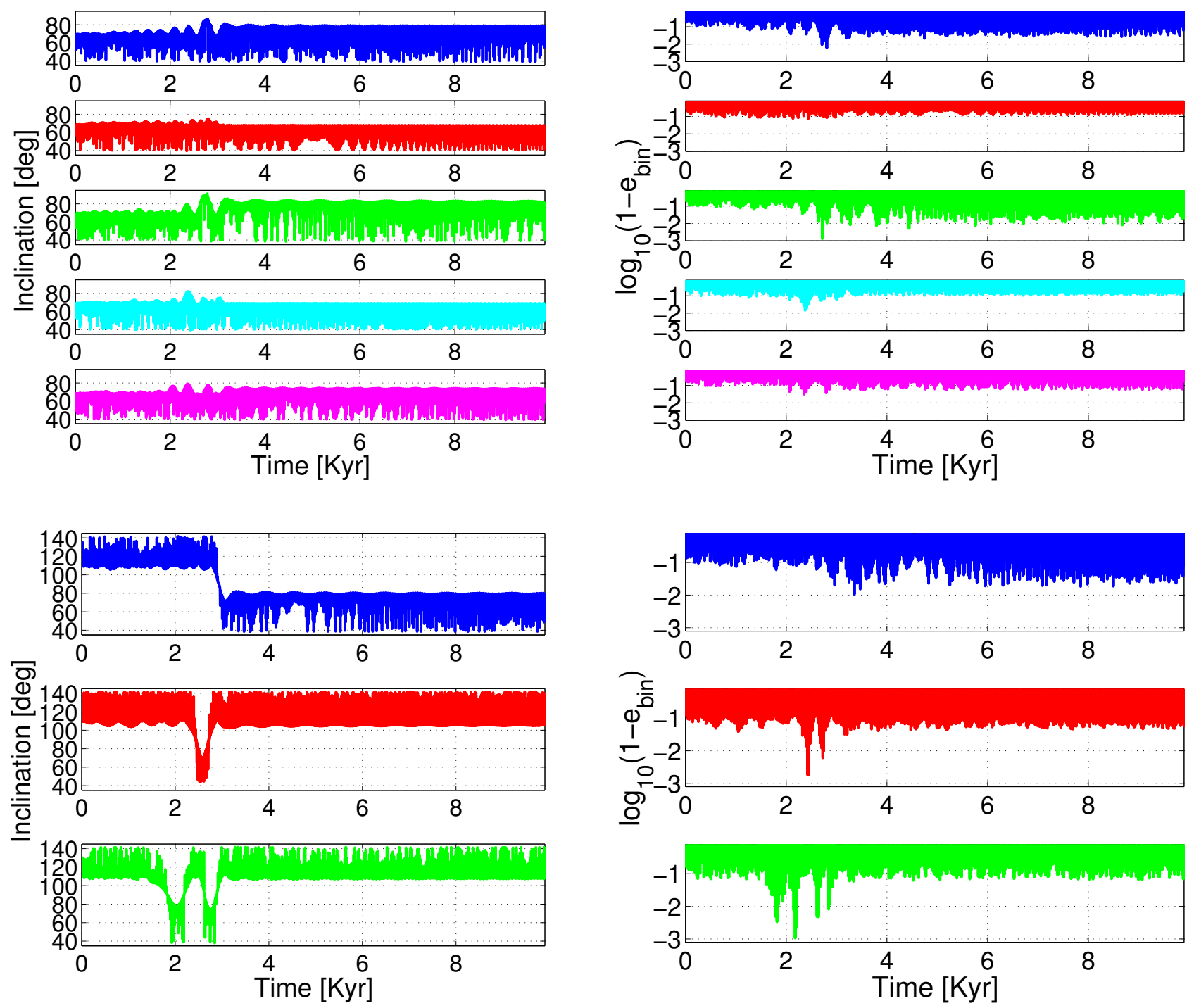

FIG. 12.- Evolution of binary orbital elements with random phase (i.e. true anomaly $\nu_{\text {bin }}$ is chosen randomly), $q=10^{-2}, Q=10^{-8}$, and outer eccentricity $e_{\text {out }}=0.1$. Top panels: $I=70 \mathrm{deg}$. Bottom panels: $I=110 \mathrm{deg}$. Left: Binary inclinations. Right: Binary eccentricity. $3 / 5$ of the retrograde binaries have survived and are presented here.

BPs (Perets \& Naoz 2009, Perets 2011). As we found in this study, evolution of BPs due to interactions with the gas (in particular due to GDF) could alter their initial distribution in the planetesimal disk, and determine the initial conditions for the later evolution once the gas has dispersed. We find that BPs on circular CM orbits have inspiraled or merged due to GDF, depending on the planetesimal-to-sun and inner binary mass ratios $Q$ and $q$, respectively. Generally, GDF is more efficient in the inner regions of the disk due to the density gradient. At $1 \mathrm{AU} \sim 10^{23} \mathrm{~g}$ mass BPs inspiral and merge within $1 \mathrm{Myr}$. If we choose the density scaling $\rho_{g} \propto a^{-16 / 7}$ (see paper I for details), the inspiral time increases by $\sim 200$ at 10AU, and only significantly affects BPs with masses $\sim 10^{25} \mathrm{~g}$. For Trans Neptunian and Kuiper Belt objects, the inspiral time is longer by a factor of a few $\times 10^{3}$, hence it can only significantly affect masses of the order of $\sim 10^{27} \mathrm{~g}$, which is much more massive than Pluto, the largest known KBO member. In addition, GDF approach does not apply to such large masses because of accretion and non-linearity of the density wake (see paper I for details). As the distance from the star increases, we expect that BPs will be more common, with larger separations and larger masses. In addition, the binary inspiral extracts internal energy and serves as an additional heat source of the gaseous and planetesimal disks.

If the external CM orbit is eccentric, the binary expands. Starting with initial separation of $0.1 r_{H}$, none of the binaries has broken up. However it is plausible that starting from larger separations, initially gravitationally bound binaries will expand, reach the limit of dynamical stability, and break. Hence, in this case a more restricted stability criteria is required. The simulated binaries have expanded roughly two times their initial separation, hence the GDF eccentric stability radius should be reduced by a factor of two. BPs with large separations have larger cross section for encounters with other planetesimals, hence they are important for the evolution of the planetesimal disk. If there is a mechanism that pumps the external eccentricity above a 
certain threshold (e.g. scattering by larger planetesimals and planets), the expansion could persist as long as the gas is present. In this case determining the break up timescale is critical to determine the fate of the BPs.

Highly inclined BPs could be excited to large eccentricities and inclinations due to KL oscillations as well as their coupling to GDF interactions. Some of them can merge, break up or flip their orientations. It is plausible that some of the highly inclined BPs survived the final stages of planet formation.

\section{SUMMARY}

In this study we extended the study of the interactions of single intermediate size planetesimals interaction with gas through gas dynamical friction (paper I) and considered the effects on binary planetesimals (BPs) in the same mass range of $10^{21}-10^{25} \mathrm{~g}$. At this mass range aerodynamic gas drag is negligible, but planetesimals have sufficiently low mass, so linear perturbation in the gas density is applicable. In addition, for such binaries the Hill radius is much smaller than the disk scale height, hence the approximation of spherical gaseous halo is more easily attained than in the single planetesimal case.

Equal mass BPs of mass $\sim 10^{23} \mathrm{~g}$ at $1 \mathrm{AU}$ and initial low orbital eccentricity inspiral in less than a Myr. This result is consistent with Perets \& Murray-Clay (2011)'s merger timescale for planetesimals of sizes of a few $\times 100 \mathrm{~km}$ BPs, close to the critical size where GDF and gas drag are comparable, and provides additional channel for mergers and inspirals for larger planetesimals. Larger planetesimals merge faster, with a general trend of $\tau_{\text {merge }} \propto m_{p}^{-1}$, that breaks down near the sonic limit where $\mathcal{M} \sim 1$.
BPs with external eccentricity $e_{\text {out }} \gtrsim 2(h / a)$, experience supersonic flow that applies a positive net torque. Such BPs gain angular momentum and expand. As their outer orbit circularizes, the torque direction is reversed, and the binary shrinks after the flow has become subsonic. Higher initial separations or persistent external eccentricity pumping could eventually lead to binary break up.

Inclined BPs are subjected to Kozai-Lidov oscillations that could affect the binary evolution. While the inspiral timescale is the same as in the co-planar case, the break up timescale is longer since the expansion is quenched. Small mass binary ratios could result in a stochastic interaction that could lead to merger, break up or inclination flip.

The interactions of BPs with gas can significantly change their evolution and thereby affect the planetesimal disk and affect the distribution of BPs and their separations, as well as induce binary merger-formed single planetesimals which could have a highly oblate structure. We conclude that GDF can play an important role in the evolution of BPs, and should be accounted for in the study of the early stages of planet formation, as well as for the origin and early evolution of current asteroids, Kuiper belt object and their binary components.

\section{ACKNOWLEDGEMENTS}

We thank Erez Michaely for stimulating discussions and the referee, Keiji Ohtsuki, for helpful comments that lead to improvement of the manuscript. HBP acknowledges support from Israel-US bi-national science foundation, BSF grant number 2012384, European union career integration grant "GRAND", the the Minerva center for life under extreme planetary conditions and the Israel science foundation excellence center I-CORE grant 1829.

\section{REFERENCES}

Baruteau, C., Cuadra, J., \& Lin, D. N. C. 2011, ApJ, 726, 28

Brown, P., \& Lawyer, D. 2003, J. Environ. Eng., 129, 222

Chiang, E. I., \& Goldreich, P. 1997, ApJ, 490, 368

Goldreich, P., Lithwick, Y., \& Sari, R. 2002, nat, 420, 643

-. 2004, araa, 42, 549

Grishin, E., \& Perets, H. B. 2015, ApJ, 811, 54

Hamilton, D. P., \& Burns, J. A. 1991, icarus, 92, 118

Hut, P., Makino, J., \& McMillan, S. 1995, ApJ, 443, L93

Kim, H., \& Kim, W.-T. 2007, ApJ, 665, 432

Kim, H., Kim, W.-T., \& Sánchez-Salcedo, F. J. 2008, ApJ, 679, L33

Kominami, J. D., \& Makino, J. 2014, PASJ, 66, 123

Kominami, J. D., Makino, J., \& Daisaka, H. 2011, PASJ, 63, 1331

Kozai, Y. 1962, AJ, 67, 591

Lidov, M. L. 1962, Planet. Space Sci., 9, 719

Murray, C. D., \& Dermott, S. F. 1999, Solar system dynamics

Muto, T., Takeuchi, T., \& Ida, S. 2011, ApJ, 737, 37
Nesvorný, D., Youdin, A. N., \& Richardson, D. C. 2010, AJ, 140, 785

Noll, K. 2007, Binaries in the Kuiper Belt: Probes of Solar System Formation and Evolution, HST Proposal

Ohtsuki, K., Nakagawa, Y., \& Nakazawa, K. 1988, icarus, 75, 552

Ohtsuki, K., Stewart, G. R., \& Ida, S. 2002, icarus, 155, 436

Ormel, C. W., \& Klahr, H. H. 2010, A\&A, 520, A43

Ostriker, E. 1999, ApJ, 513, 252

Papaloizou, J. C. B., \& Terquem, C. 2006, Reports on Progress in Physics, 69, 119

Perets, H. B. 2011, ApJ, 727, L3

Perets, H. B., \& Murray-Clay, R. A. 2011, ApJ, 733, 56

Perets, H. B., \& Naoz, S. 2009, ApJ, 699, L17

Pfalzner, S., Steinhausen, M., \& Menten, K. 2014, ApJ, 793, L34

Richardson, D. C., \& Walsh, K. J. 2006, Annual Review of Earth and Planetary Sciences, 34, 47

Sánchez-Salcedo, F. J., \& Brandenburg, A. 1999, ApJ, 522, L35

Sánchez-Salcedo, F. J., \& Chametla, R. O. 2014, ApJ, 794, 167

\section{APPENDIX}

\section{A. BINARY STABILITY}

The Hill radius is defined as the distance at which the gravitational tidal acceleration equals the gravitational acceleration of the binary. Similarly to the definition of Hill radius, the GDF shearing radius is defined as the the distance at which the gravitational tidal acceleration equals the magnitude of the differential acceleration $\Delta a_{G D F}$. Equating of the gravitational acceleration $a_{\text {grav }}=G\left(m_{b}+m_{s}\right) / a_{b i n}^{2}$ to the shearing acceleration yields the GDF shearing radius

$$
R_{G D F}=\sqrt{\frac{G\left(m_{b}+m_{s}\right)}{\Delta a_{G D F}}} .
$$


Taking $\mathcal{A}_{c m}$ and $\mathcal{A}_{b i n}$ from Eqn. 18$)$, the magnitude of the differential acceleration is $\left|\boldsymbol{\Delta} \boldsymbol{a}_{\boldsymbol{G} \boldsymbol{D \boldsymbol { D F }}}\right|=\left(\mathcal{A}_{c m}^{2}+\mathcal{A}_{b i n}^{2}+\right.$ $\left.2 \mathcal{A}_{c m} \mathcal{A}_{\text {bin }} \hat{\boldsymbol{\varphi}} \cdot \hat{\varphi}_{\text {bin }}\right)^{1 / 2} \leq 2 \mathcal{A}$, where $\mathcal{A} \equiv \max \left\{\mathcal{A}_{c m}, \mathcal{A}_{\text {bin }}\right\}$. The GDF shearing radius is bounded from below by

$$
R_{G D F} \geq \sqrt{\frac{G m_{b i n}}{2 \mathcal{A}}} .
$$

For each regime, the linear and the supersonic, there are two distinct cases: equal and very small mass ratio binaries. For Linear regime and equal mass binaries, $q=1, m_{s}=m_{b}=m$ and $\mathcal{A}_{c m}=0$, at $1 \mathrm{AU} c_{s, 1 A U}=6.7 \cdot 10^{4} \mathrm{~cm} / \mathrm{s}$

$$
\begin{aligned}
R_{G D F, \text { linear }}^{\text {equal }} & =\sqrt{\frac{G m_{\text {bin }}}{\mathcal{A}_{\text {bin }}}}=\sqrt{\frac{3 c_{s}^{3}}{2 \pi G \rho v_{\text {bin }}}} \\
& =\sqrt{\frac{3 H_{0}}{2 \pi}} f^{1 / 4} Q^{-1 / 6} \frac{c_{s}}{\sqrt{G \rho}} \\
& =0.84\left(\frac{H_{0}}{0.022}\right)^{1 / 2}\left(\frac{f}{0.1}\right)^{1 / 4}\left(\frac{Q}{10^{-10}}\right)^{-1 / 6}\left(\frac{c_{s}}{c_{s, 1 A U}}\right) A U .
\end{aligned}
$$

For small mass binary, $q \ll 1, \mathcal{A}_{c m} \gg \mathcal{A}_{\text {bin }}$ we have

$$
\begin{aligned}
R_{G D F, \text { linear }}^{\text {small }} & \approx \sqrt{\frac{G m_{\text {bin }}}{\mathcal{A}_{c m}}}=\sqrt{\frac{1+q}{4 \pi H_{0}(1-q)}} \frac{c_{s}}{\sqrt{G \rho}} \\
& \approx 0.59\left(\frac{H_{0}}{0.022}\right)\left(\frac{c_{s}}{c_{s, 1 A U}}\right) A U .
\end{aligned}
$$

The correction for Eqn $(\mathrm{A} 2)$ is of order $\mathcal{O}(q)$. In this case, the shearing radius is smaller by $\sim 70 \%$ compared to the equal mass case. We expect that in the general case where $\mathcal{A}_{c m}$ and $\mathcal{A}_{\text {bin }}$ are comparable, the result will be somewhere between these two extreme values.

For the supersonic regime and small $q$, the first term in Eqn $(17)$ is the dominant one, and the GDF shearing radius is

$$
\begin{aligned}
R_{G D F, \text { super }}^{\text {small }} & =\sqrt{\frac{(1+q) G e_{p}^{2} v_{K}^{2}}{(1-q) C_{\text {super }}}}=\sqrt{\frac{(1+q) e_{p}^{2} v_{K}^{2}}{(1-q) 4 \pi G \rho_{g} \ln \Lambda}} \\
& \approx 1.07 e_{p} R_{G D F, \text { linear }}^{\text {small }}
\end{aligned}
$$

The latter result is valid only when the supersonic regime applies, i.e. $e \gtrsim 2 H_{0} \approx 0.044$, so the minimal GDF shearing radius is $\sim 20$ times smaller, but still much larger than the Hill radius.

For equal mass binaries, the second and third term is in Eqn (17) are comparable, and the GDF shearing radius is

$$
R_{G D F, \text { super }}^{\text {equal }} \approx \frac{1}{2} \sqrt{\frac{2 G e_{p}^{3} v_{K}^{3}}{C_{\text {super }} 3 v_{b i n}}}=\frac{1}{2} \sqrt{\frac{e_{p}^{2} v_{K}^{2}}{6 \pi G \rho_{g} \ln \Lambda \beta}} \approx R_{G D F, \text { super }}^{\text {small }} \sqrt{\frac{1}{6 \beta}} .
$$

Since $\beta$ is small, the GDF shearing radius is larger.

In summary, in all the cases we considered, the GDF shearing radius is always much larger than the Hill radius, therefore the binaries are stable to perturbations from GDF.

\section{B. DERIVATION OF EQUATION 17}

Starting from eqn 16, expanding to first order in $\beta$, rearranging terms and using the definition of the reduced mass $\mu$, as well as replacing $\beta$ back to $v_{\text {bin }}$ leads to

$$
\begin{aligned}
\frac{\boldsymbol{\Delta} \boldsymbol{a}_{G D F}}{C_{\text {super }}} & =\frac{1}{e_{\text {out }}^{2} v_{K}^{2}}\left[\left(m_{b} \hat{\boldsymbol{\varphi}}-m_{b} \frac{m_{s}}{m_{\text {bin }}} \beta \hat{\boldsymbol{\varphi}}_{\text {bin }}\right)\left(1+3 \frac{m_{s}}{m_{\text {bin }}} \beta\right)-\left(m_{s} \hat{\boldsymbol{\varphi}}+m_{s} \frac{m_{b}}{m_{\text {bin }}} \beta\right) \hat{\boldsymbol{\varphi}}_{\text {bin }}\left(1-3 \frac{m_{s}}{m_{\text {bin }}} \beta\right)\right] \\
& =\frac{m_{b}\left(e_{\text {out }} v_{K}(1-q)+3 q v_{\text {bin }}\right) \hat{\boldsymbol{\varphi}}-2 \mu v_{\text {bin }} \hat{\boldsymbol{\varphi}}_{\text {bin }}}{e_{\text {out }}^{3} v_{K}^{3}}
\end{aligned}
$$

